\title{
HETEROSIS AND COMBINING ABILITY FOR SOME FRUIT QUALITY TRAITS OF EGYPTIAN MELON INBRED LINES USING LINE $\times$ TESTER ANALYSIS
}

\author{
M.A.M. SELIM \\ Vegetable, Medicinal and Aromatic Plants Breeding Research Department, \\ Hort. Res. Institute, ARC, Giza, Egypt.
}

(Manuscript received 25 March 2019)

\begin{abstract}
$\mathrm{T}$ his study was carried out during the period from 2016 to 2018 summer seasons in the open field at Kaha Vegetable Research Farm (KVRF), Kalubia, Egypt. Thirty local inbred lines of melon (Cucumis melo var. ananas) were evaluated in this investigation during 2016 and 2017 summer seasons in the open field to determine their mean performance. Based on the former evaluation, 12 inbred lines (RIL A2, RIL A3, RIL A7, RIL A8, RIL A14, RIL A18, RIL A19, RIL A22, RIL A23, RIL A26, RIL A29, RIL A30) and 3 testers (RIL A5, RIL A10 and RIL A20) were selected to determine their genetic performance using line $\times$ tester mating design during 2018 summer season. Thirty six crosses in one direction were conducted in the plastic house of Kaha Vegetable Research Farm, Kalubia through the 2017 late summer season. The 36 hybrids were evaluated along with their parents to determine heterosis, combining ability and heritability of leaf area index (LAI), yield components, average fruit weight, netting percentage, fruit shape index (FSI), seed cavity diameter, flesh thickness and total soluble solids (TSS). The genotype results showed highly significant mean squares for most of the studied traits. Inbred lines (female parents), Testers (male parents) and Line $x$ Tester interaction showed highly significant differences of almost whole traits. Some crosses revealed highly significant and significant mid-parent and better-parent heterosis for many of the traits. The inbred line RIL A5 (T5) showed higher positive general combining ability (GCA) impact for all traits except early yield and FSI, which could be used as parent in breeding programs and the potential parent (good combiner) that in selection program would be effective for its efficient use in subsequent crossing programs for more LAI, total yield, marketable yield percentage, average fruit weight, netting percentage, flesh thickness, TSS and less seed cavity diameter. Nine, eight, twelve and seven hybrids revealed highly significant and significant specific combining ability (SCA) impacts of early yield, total yield, average fruit weight and TSS, respectively. The best specific combining ability (SCA) was observed in hybrids RIL A29 $\times$ RIL A5 for early yield, RIL A19 $\times$ RIL A20 for total yield and RIL A8 $\times$ RIL A10 for average fruit weight and TSS. The results confirmed the presence of genetic differences among the genotypes (female and male parents) and heterosis of crosses indicating the existence of predominance of nonadditive gene action in genetic control of the studied traits.
\end{abstract}

Key Words: Melon, Line $\times$ Tester analysis, Heterosis, Combining ability, heritability. 


\section{INTRODUCTION}

Melon (Cucumis melo L.) is a cross-pollinated plant and an economically important crop species of Curcubitaceae family. Breeding strategies depend on selection of the best hybrids which need strong level of heterosis along with the specific combining ability. In classic breeding the great yielding cultivars of any crop, the breeders usually meet with the obstacle of screening parents and crosses. One of the strongest tools for determining the combining ability impacts is combining ability analysis which helps in choosing the best parents and crosses for the exploitation of heterosis. Line $x$ tester analysis determines the various types of gene actions by giving information about general combining ability (GCA) and specific combining ability (SCA) effects of parents (Spargue and Tatum, 1942; Griffing, 1956 and Shashikumar and Pitchaimuthu, 2016).

Heterosis breeding is one of the most efficient tool of plant breeders to exploit the genetic diversity (Chaudhary and Pandey, 2010). Munger (1942) was the first to observe hybrid vigour in melon. Melon is enriched with great variability and therefore, heterosis breeding can be efficiently utilized to produce hybrids containing high yield and fruit quality (Pandeyet al., 2005). Robinson et al. (1976) observed variability in vine length (1-10 m), fruit weight (10 g -10 Kg), TSS (3-18\%) and flesh acidity (pH 3-7) in melon. Moreover, there is a need to develop suitable hybrids, which may be utilized on commercial scale (Dhaliwal, 1997).

Moreand Seshadri (1998), Peter and Swamy (2006) and Pitrat (2009) advocated the following breeding goals for melon breeding programme, viz., great yield and regular fruit shape and size, are determinants for superior melon hybrids. Likewise, an early and hard netted skin fruit having narrow seed cavity are the important traits. Being dessert fruit, quality parameters, especially TSS, flesh thickness, texture and colour should be taken into consideration. The total soluble solids content should vary from $11-13 \%$, but not less than $10 \%$. Also, the hybrid should be resistance to biotic stresses.

Heterobeltiosis for fruit weight, flesh thickness, total yield and TSS content was observed in melon by Chadha and Nandpuri (1980), Dixit and Kalloo (1983), Mishra and Seshadri (1985), Randhawa and Singh (1990), Dhaliwal and Lal (1996), Munshi and Verma (1997), Lal and Kaur (2002), Chaudharyet al. (2003) and Subramanian (2008).

Usually, heterosisis contributing a great yield for definite cultivars. The breeding efforts made increasing in quality and percentage of marketable yield over total yield in different vegetable crops (Gusmini and Wehner, 2008). Development of fruit quality traits (especially TSS) by selection is very hard due to it is very effective by the 
environmental factors; so, it isn't possible to improve it within architectural limits even in pure varieties. Since its genetic and expression is very complicated, so it's essential to know whether $F_{1}$ hybrids have superior to the pure bred varieties and heterozygosity may lead to the genetic equilibrium necessary in the appearance of this quality attributes (More and Seshadri, 1998).

Total fruit weight, single fruit weight, fruit length and fruit shape in melon showed variable levels of best parent heterosis ranging from highly positive to negative with some differences among the trials, while total soluble solids displayed mainly additive phenotypic effect, although with large variability across trials. Fruit diameter displayed mainly dominant phenotypic effect and earliness showed large differences between locations, suggesting that it greatly depends on the environment (Napolitanoet al., 2017). Also, Heterosis values were significant over better parent for growth, earliness and yield characters in melon (Duradundiet al., 2018). The broad and narrow sense heritability in melon were low for average fruit weight, flesh thickness and total yield, but they were high for TSS (Mohammadiet al., 2014).In contrast, Javanmard et al. (2018) found that narrow sense heritability was high for all melon traits except fruit diameter and TSS. These results indicate that selection may be more effective for improving traits of genotypes in early generations.

Several authors reported that additive and non-additive effects in the genetic control of the fruit weight in melon (Lippert and Legg, 1972; Kalb and Davis, 1984; Singh and Randhawa, 1990; Monforte et al., 2004). Feyzianet al. (2009) found that average fruit weight was controlled by additive effects in a diallel of local melon populations in Iran. Lippert and Legg (1972) studied the gene action of yield trait in melon and stated that additive and non-additive variance components were important in the genetic control of yield correlated characters.

As the efforts in heterosis breeding are inadequate, the area under F1 hybrids in muskmelon is very negligible in Egypt. Most essential steps in this direction is identification of superior heterotic F1 hybrids for yield, quality and earliness. General and specific combing ability for quantitative characters manipulating yield and its components is very beneficial in screening parents for production of superior hybrids (Duradundi et al. 2018).

So, the present study aimed to determine the combining ability effects of some melon parents and hybrids for different traits and estimating the magnitude of heterobeltiosis, broad and narrow sense heritability in the hybrids. 


\section{MATERIALS AND METHODS}

This study was carried out during the period from 2016 to 2018 summer seasons at Kaha Vegetable Research Farm (KVRF), Kalubia, Egypt, in the open field using a drip-irrigation system and polyethylene plastic mulch. Thirty local inbred lines of melon (Cucumis melo var. ananas) were used in this investigation. These inbred lines were originated by the author of the present study from former melon breeding program by selfing and selection during 12 generations.

Based on data obtained from the thirty inbred lines that were evaluated during 2016 and 2017 summer seasons, the 12 inbred lines and 3 testers were selected to determine their genetic performance using line $\times$ tester mating design during 2018 summer season. 36 crosses in one direction were conducted in the plastic house facilities of Kaha Vegetable Research Farm, Kalubia during the 2017 late summer season. The 36 hybrids were evaluated along with their parents (12 inbred lines as female parent and 3 testers as male parent) during 2018 summer season in the open field.

Seeds of the 30 inbred lines evaluation and line $\times$ tester experiment were sown on 5 March, 2016, 2017and 2018, respectively, in foam trays under greenhouse. In a randomized complete block design with 3 replicates, Seedlings were transplanted on April 1st in the open field. Thirty experimental plots (EP) of each replicate to evaluate the inbred lines in 2016 and 2017. Also in the line $\times$ tester experiment, 51 experimental plots (12inbred lines, 3 testers and $36 F_{1}$ s) of each replicate were evaluated during 2018 summer season. A single bed dimensions were $1.5 \mathrm{~m}$ width and $8.0 \mathrm{~m}$ length ( $E P$ area $=12 \mathrm{~m}^{2}$ ) of each plot and the plants were sown at $50 \mathrm{~cm}$. Land preparation, fertilizer application and other field practices were conducted according to recommendations of the Egyptian Ministry of Agriculture.

The measured traits of all treatments were as following:-

1. Leaf area index ( LAI ) : The area meter ( LI-COR, model: LI 3050A/4,U.S.A) was used to determine the leaf area of each plant after fruits maturity. An average of 5 randomly chosen plants was measured per EP and the average leaf area was divided by the ground area occupied by the plant to calculate the LAI.

2. Yield: The yield of the first 3 pickings was measured to determine the early yield (EY) as, the weight of all fruits harvested at the yellow-netted ripe stage from each EP was measured to determine the total yield (TY). Marketable yield (MY) as determined after excluding cracked, rotten and infected fruits with diseases and pests and was calculated as percentage from the total yield.

3. Fruit quality: average fruit weight (AFW), seed cavity diameter and flesh thickness were measured as the average of 15 fruits randomly taken from each EP, 
fruit shape index (FSI) computed as the ratio of fruit length to fruit diameter. Each EP was represented by 15 fruits. Fruits with a FSI below 0.88 were defined as oblate, those with a FSI limited to 0.88 and 1.1 were reported round, those with a FSI limited to 1.1 to 1.5 were defined as cylindrical and those with a FSI over 1.5 were defined as oblong (Rashidi and Seyfi 2007). The netting percentage was estimated as a proportion of the netting coated fruit cortex to full fruit cortex as optical manner and calculated as the average of 15 fruits randomly taken from each EP. Total soluble solids (TSS) was measured in 15 yellow-ripe fruits of each EP utilizing a hand refractometer. Finally, the fruit flesh colour was determined as descripted method by naked eye to identify the flesh colour.

Collected data were statistically analyzed and mean comparisons were depend on the LSD test as reported by Gomez and Gomez (1984). Also, the Bartlett's test (utilizing Chi-square test) of the variance of errors for both years (2016 and 2017) were homogeneous for all traits. So, the combined analysis of variance for both years was calculated for all traits as reported by Koch and Sen (1968).

The data were displayed combining ability analysis as stated in Kempthorne (1957). Heterosis was determined as per method suggested by Bitzeret al. (1967) and Wynne et al. (1970). Heterosis over mid parent and better parent was estimated as percentage after calculating heterosis of respective parent by utilizing formula as reported by Falconer and Mackay (1996).

\section{RESULTS AND DISCUSSION}

\section{Evaluation of inbred lines}

\section{Leaf Area Index (LAI)}

Obtained data on LAI and Yield in the summer seasons of 2016 and 2017 were combined in Table (1).

LAI data showed that RIL A20 had the highest LAI and was significantly different from whole the others. RIL A1 sorted second in this trait, but it wasn't significantly different from RIL A5. In contrast, RIL A11 had the least LAI, but it wasn't significantly different from RILs A12, A23 and A28. 
Table 1. Leaf area index and yield of some ananas melon RILs evaluated during the combined 2016 and 2017 summer seasons.

\begin{tabular}{|c|c|c|c|c|}
\hline Inbred line & Leaf area index & $\begin{array}{c}\text { Early yield } \\
\text { (ton / feddan) }\end{array}$ & $\begin{array}{c}\text { Total yield } \\
\text { (ton / feddan) }\end{array}$ & $\begin{array}{c}\text { Marketable yield } \\
\text { (\%) }\end{array}$ \\
\hline RIL A1 & $1.35 \mathrm{ghi}$ & $1.05 \mathrm{ghi}$ & 11.71 efghi & $71.10 \mathrm{i}$ \\
\hline RIL A2 & $1.23 \mathrm{hij}$ & $1.47 \mathrm{de}$ & 12.76 cdef & 91.26 abcde \\
\hline RIL A3 & $1.59 \mathrm{cde}$ & $1.86 \mathrm{c}$ & $13.89 \mathrm{c}$ & $93.89 \mathrm{abcd}$ \\
\hline RIL A4 & $1.35 \mathrm{ghi}$ & $0.91 \mathrm{hij}$ & $10.43 \mathrm{jk}$ & $82.44 \mathrm{gh}$ \\
\hline RIL A5 & $2.47 \mathrm{~b}$ & $0.73 \mathrm{jkl}$ & $18.84 \mathrm{a}$ & $96.13 \mathrm{a}$ \\
\hline RIL A6 & $1.11 \mathrm{jk}$ & $0.49 \mathrm{klm}$ & $8.44 \mathrm{mno}$ & $63.41 \mathrm{k}$ \\
\hline RIL A7 & $1.09 \mathrm{jk}$ & $1.47 \mathrm{de}$ & $11.88 \mathrm{defg}$ & $93.45 \mathrm{abcd}$ \\
\hline RIL A8 & $1.50 \mathrm{efg}$ & $2.04 \mathrm{c}$ & 12.93 cde & 91.77 abcde \\
\hline RIL A9 & 1.59 cde & $0.61 \mathrm{klm}$ & 10.93 ghijk & $86.95 \mathrm{efg}$ \\
\hline RIL A10 & $2.56 \mathrm{~b}$ & $3.22 \mathrm{a}$ & $16.70 \mathrm{~b}$ & $96.15 \mathrm{a}$ \\
\hline RIL A11 & 0.891 & $0.76 \mathrm{ijk}$ & 7.76 no & $73.60 \mathrm{i}$ \\
\hline RIL A12 & 0.931 & $1.11 \mathrm{fgh}$ & $10.54 \mathrm{ijk}$ & $82.68 \mathrm{gh}$ \\
\hline RIL A13 & $1.24 \mathrm{hij}$ & $0.55 \mathrm{klm}$ & $8.91 \mathrm{mn}$ & $70.08 \mathrm{i}$ \\
\hline RIL A14 & $1.17 \mathrm{jk}$ & $1.99 \mathrm{c}$ & $12.71 \mathrm{cdef}$ & 89.04 cdef \\
\hline RIL A15 & $1.69 \mathrm{~cd}$ & $0.44 \mathrm{~lm}$ & $12.11 \mathrm{defg}$ & $84.02 \mathrm{fgh}$ \\
\hline RIL A16 & $1.72 \mathrm{c}$ & $1.22 \mathrm{efg}$ & $10.33 \mathrm{jkl}$ & 90.30 bcde \\
\hline RIL A17 & $1.19 \mathrm{jk}$ & $0.33 \mathrm{~m}$ & $8.87 \mathrm{mn}$ & $72.06 \mathrm{i}$ \\
\hline RIL A18 & $1.53 \mathrm{ef}$ & $2.04 \mathrm{c}$ & $10.49 \mathrm{ijk}$ & 94.00 abc \\
\hline RIL A19 & $1.48 \mathrm{efg}$ & $1.17 \mathrm{fgh}$ & $12.98 \mathrm{~cd}$ & 88.68 def \\
\hline RIL A20 & $2.77 \mathrm{a}$ & $1.35 \mathrm{def}$ & $15.71 \mathrm{~b}$ & 90.58 bcde \\
\hline RIL A21 & $1.18 \mathrm{jk}$ & $0.69 \mathrm{jkl}$ & $9.15 \mathrm{Im}$ & $64.51 \mathrm{jk}$ \\
\hline RIL A22 & $1.53 \mathrm{ef}$ & $2.13 \mathrm{c}$ & $10.16 \mathrm{kl}$ & $83.98 \mathrm{fgh}$ \\
\hline RIL A23 & $1.04 \mathrm{kl}$ & $1.52 \mathrm{~d}$ & 10.64 hijk & $92.90 \mathrm{abcd}$ \\
\hline RIL A24 & $1.22 \mathrm{ij}$ & 1.19 efgh & $8.63 \mathrm{mno}$ & $69.15 \mathrm{ij}$ \\
\hline RIL A25 & $1.38 \mathrm{fgh}$ & $0.45 \mathrm{~lm}$ & 7.610 & $80.87 \mathrm{~h}$ \\
\hline RIL A26 & $1.70 \mathrm{~cd}$ & $2.50 \mathrm{~b}$ & $8.45 \mathrm{mno}$ & $94.48 a b$ \\
\hline RIL A27 & $1.56 \mathrm{de}$ & $0.55 \mathrm{klm}$ & $8.24 \mathrm{mno}$ & $73.66 \mathrm{i}$ \\
\hline RIL A28 & $1.04 \mathrm{kl}$ & $1.21 \mathrm{efg}$ & $12.75 \mathrm{cdef}$ & $62.66 \mathrm{k}$ \\
\hline RIL A29 & $1.69 \mathrm{~cd}$ & $1.96 \mathrm{c}$ & $11.53 \mathrm{fghij}$ & $92.92 \mathrm{abcd}$ \\
\hline RIL A30 & $1.15 \mathrm{jk}$ & $1.52 \mathrm{~d}$ & 11.81 defgh & $93.04 \mathrm{abcd}$ \\
\hline LSD & 0.16 & 0.30 & 1.24 & 5.27 \\
\hline
\end{tabular}




\section{Yield and its components}

\section{a) Early Yield}

The RIL A10 had the greatest EY and was significantly different from whole the others. RIL A26 ranked second in this trait and was significantly different over all other evaluated RILs. Also, RIL A22 ranked third in this trait without significant differences from RILs A18, A8, A14, A29 and A3. On the other hand, RIL A17 produced the least $E Y$ with non-significant differences from RILs $A 15, A 25, A 6, A 27$, A13 and A9.

\section{b) Total Yield}

The greatest TY was shown in the RIL A5 and it was significantly different over all other evaluated RILs. Additionally, RILs A10 and A20 ranked second in this trait. In contrast, RIL A25 had the least TY with non-significant differences from RILs A11, A27, A26, A24 and A6.

\section{c) Marketable Yield}

The RIL A10 produced the highest MY (\%) without significant differences from RILs A5, A2, A3, A7, A8, A18, A23, A26, A29 and A30. Additionally, RIL A26 ranked second in this trait but it wasn't significantly different from RILs A18, A3, A2, A7, A8, $A 16, A 20, A 23, A 29$ and $A 30$. In contrast, RIL A28had the least MY(\%)with nonsignificant differences from RILs A6 and A21.

\section{Fruit Quality}

Obtained data on AFW, FSI and netting (\%)in the summer seasons of 2016 and 2017 were combined in Table(2).

Regarding AFW, the RIL A20 gave the highest AFW and was significantly different from whole the others. The RIL A10 sorted second but it wasn't significantly different from RILs A5 and 14. The least AFW was shown in RIL A23 but it wasn't significantly different from RILsA4, A6, A7, A8, A12 and A16. Concerning fruit shape index (FSI), the RIL A26 had the highest FSI with significant different over all other evaluated RILs. Also, the RIL A16 ranked second in this trait. The least FSI was shown in RIL A1 with no significant different from RIL A25. In respect to netting percentage, the RIL A1 had the highest netting percentage without significant differences from the most evaluated RILs. In addition, the RIL A4 ranked second in this trait without significant differences from RIL A12. The least netting percentage was shown in RIL A18 with no significant different from RILs A24, A27 and A28. 
Table 2. Average fruit weight, fruit shape index and netting percentage of some ananas melon RILs evaluated during the combined 2016 and 2017 summer seasons.

\begin{tabular}{|c|c|c|c|}
\hline Inbred line & $\begin{array}{l}\text { Average fruit weight } \\
(\mathrm{Kg})\end{array}$ & Fruit shape index & $\begin{array}{c}\text { Netting } \\
(\%)\end{array}$ \\
\hline RIL A1 & $1.15 \mathrm{kImno}$ & $1.02 \mathrm{~s}$ & $100.00 \mathrm{a}$ \\
\hline RIL A2 & 1.32 ghijkl & 1.270 & $60.13 \mathrm{~g}$ \\
\hline RIL A3 & 1.38 efghij & $1.19 \mathrm{p}$ & $68.25 \mathrm{f}$ \\
\hline RIL A4 & 1.06 mnop & $1.35 \mathrm{n}$ & $87.75 \mathrm{~b}$ \\
\hline RIL A5 & $1.68 \mathrm{bc}$ & $1.57 \mathrm{ij}$ & $100.00 \mathrm{a}$ \\
\hline RIL A6 & 1.04 nop & $1.64 \mathrm{~h}$ & $100.00 \mathrm{a}$ \\
\hline RIL A7 & 1.03 nop & $1.80 \mathrm{e}$ & $84.50 \mathrm{c}$ \\
\hline RIL A8 & 1.12 Imnop & $1.48 \mathrm{Im}$ & $100.00 \mathrm{a}$ \\
\hline RIL A9 & $1.18 \mathrm{jkImn}$ & $1.66 \mathrm{gh}$ & $73.13 \mathrm{e}$ \\
\hline RIL A10 & $1.79 \mathrm{~b}$ & $1.59 \mathrm{i}$ & $100.00 \mathrm{a}$ \\
\hline RIL A11 & $1.16 \mathrm{klmno}$ & $1.97 \mathrm{c}$ & $79.63 \mathrm{~d}$ \\
\hline RIL A12 & 1.14 klmnop & $1.89 \mathrm{~d}$ & $87.75 b$ \\
\hline RIL A13 & 1.46 defgh & 1.250 & $100.00 \mathrm{a}$ \\
\hline RIL A14 & $1.60 \mathrm{bcd}$ & 1.78 ef & $100.00 \mathrm{a}$ \\
\hline RIL A15 & 1.38 efghij & $1.47 \mathrm{~m}$ & $60.13 \mathrm{~g}$ \\
\hline RIL A16 & 0.96 op & $2.10 \mathrm{~b}$ & $74.75 \mathrm{e}$ \\
\hline RIL A17 & 1.38 efghij & $1.27 \mathrm{o}$ & $81.25 d$ \\
\hline RIL A18 & 1.33 fghijk & $1.12 \mathrm{q}$ & $0.00 \mathrm{j}$ \\
\hline RIL A19 & $1.23 \mathrm{ijklmn}$ & $1.88 \mathrm{~d}$ & $45.50 \mathrm{i}$ \\
\hline RIL A20 & $2.31 \mathrm{a}$ & $1.50 \mathrm{~lm}$ & $100.00 \mathrm{a}$ \\
\hline RIL A21 & 1.54 cdef & $1.95 \mathrm{c}$ & $100.00 \mathrm{a}$ \\
\hline RIL A22 & 1.42 defghi & $1.54 \mathrm{jk}$ & $79.63 \mathrm{~d}$ \\
\hline RIL A23 & $0.93 p$ & $1.69 \mathrm{~g}$ & $53.63 \mathrm{~h}$ \\
\hline RIL A24 & $1.55 \mathrm{cde}$ & $1.07 \mathrm{r}$ & $0.00 \mathrm{j}$ \\
\hline RIL A25 & 1.38 efghij & $1.03 \mathrm{~s}$ & $100.00 \mathrm{a}$ \\
\hline RIL A26 & $1.49 \mathrm{cdefg}$ & $2.31 \mathrm{a}$ & $61.75 \mathrm{~g}$ \\
\hline RIL A27 & $1.23 \mathrm{ijklmn}$ & $1.75 \mathrm{f}$ & $0.00 \mathrm{j}$ \\
\hline RIL A28 & 1.40 defghi & $1.51 \mathrm{kl}$ & $0.00 \mathrm{j}$ \\
\hline RIL A29 & 1.26 hijklm & $1.81 \mathrm{e}$ & $61.75 \mathrm{~g}$ \\
\hline RIL A30 & 1.22 ijklmn & $1.37 \mathrm{n}$ & $100.00 \mathrm{a}$ \\
\hline LSD & 0.21 & 0.04 & 2.25 \\
\hline
\end{tabular}

Also, obtained data on seed cavity diameter, flesh thickness, TSS and flesh colour in the summer seasons of 2016 and 2017 were combined in Table (3).

Concerning seed cavity diameter, the RIL A10 had the least seed cavity diameter with significant difference over all other evaluated RILs. Also, the RIL A5 ranked second in this trait without significant different from RILs A20, A22, A25 and A26. In contrast, the largest seed cavity diameter was shown in RIL A2 with significant difference over all other evaluated RILs. Regarding flesh thickness, the RIL A20 had the greatest flesh thickness with significant difference over all other 
evaluated RILs. The RIL A10 ranked second in this trait with no significant different from RIL A5. In contrast, the narrowest flesh thickness was shown in RIL A28 without significant difference from RIL A21. In respect to total soluble solids (TSS), the RILA10 had the highest TSS without significant different from RILs A20 and A5. Additionally, the RIL A29 ranked second in this trait without significant difference from the most of other evaluated RILs. On the other hand, the RIL A26 had the lowest TSS with no significant different from RIL A21. Concerning the flesh colour, the most of evaluated RILs had cream flesh colour besides the rest had green flesh colour and orange flesh colour.

Table 3. Seed cavity diameter, flesh thickness, total soluble solids and flesh colour of some ananas melon RILs evaluated during the combined 2016 and 2017 summer seasons.

\begin{tabular}{|c|c|c|c|c|}
\hline Inbred line & $\begin{array}{l}\text { Seed cavity diameter } \\
(\mathrm{cm})\end{array}$ & $\begin{array}{c}\text { Flesh thickness } \\
(\mathrm{cm})\end{array}$ & $\begin{array}{c}\text { Total soluble } \\
\text { solids } \\
(\%)\end{array}$ & Flesh colour \\
\hline RIL A1 & $5.65 \mathrm{fgh}$ & $2.82 \mathrm{ijk}$ & 8.47 jklm & green \\
\hline RIL A2 & $7.06 \mathrm{a}$ & 3.16 fghi & 8.98 hijk & cream \\
\hline RIL A3 & $5.13 \mathrm{ijk}$ & 2.87hijk & 9.52 efghi & cream \\
\hline RIL A4 & $4.89 \mathrm{kl}$ & 3.23efgh & $8.71 \mathrm{ijkl}$ & green \\
\hline RIL A5 & $4.44 \mathrm{~m}$ & $4.31 \mathrm{~b}$ & $12.89 \mathrm{a}$ & orange \\
\hline RIL A6 & $5.10 \mathrm{ijk}$ & 2.251 & $8.56 \mathrm{ijklm}$ & cream \\
\hline RIL A7 & $6.11 \mathrm{~cd}$ & $3.66 \mathrm{~cd}$ & 9.38 ghij & cream \\
\hline RIL A8 & $5.10 \mathrm{ijk}$ & $3.26 \mathrm{efg}$ & $11.05 \mathrm{bc}$ & cream \\
\hline RIL A9 & $5.22 \mathrm{ijk}$ & 2.161 & $8.34 \mathrm{klm}$ & green \\
\hline RIL A10 & $3.95 \mathrm{n}$ & $4.45 \mathrm{~b}$ & $13.73 \mathrm{a}$ & cream \\
\hline RIL A11 & $6.45 b c$ & $2.73 \mathrm{jk}$ & 10.53 cde & cream \\
\hline RIL A12 & 5.30 hij & 3.39 def & $7.56 \mathrm{mn}$ & cream \\
\hline RIL A13 & $4.98 \mathrm{jk}$ & 3.23efgh & $11.41 b c$ & orange \\
\hline RIL A14 & $5.71 \mathrm{efg}$ & $2.72 \mathrm{jk}$ & 10.46 cdef & green \\
\hline RIL A15 & $5.69 \mathrm{fg}$ & $2.63 \mathrm{k}$ & 9.05 hijk & orange \\
\hline RIL A16 & 5.96 def & $3.29 \mathrm{defg}$ & $7.83 \mathrm{Im}$ & cream \\
\hline RIL A17 & $5.16 \mathrm{ijk}$ & $3.31 \mathrm{defg}$ & $10.67 \mathrm{bcd}$ & green \\
\hline RIL A18 & $5.09 \mathrm{ijk}$ & $3.82 \mathrm{c}$ & $8.51 \mathrm{ijklm}$ & cream \\
\hline RIL A19 & $6.58 \mathrm{~b}$ & 2.95 ghijk & 10.40 cdefg & orange \\
\hline RIL A20 & $4.51 \mathrm{~m}$ & $5.30 \mathrm{a}$ & $13.64 \mathrm{a}$ & orange \\
\hline RIL A21 & $5.23 \mathrm{ijk}$ & $1.98 \mathrm{Im}$ & 6.55 no & cream \\
\hline RIL A22 & $4.58 \mathrm{Im}$ & $3.56 \mathrm{cde}$ & 9.89 defgh & cream \\
\hline RIL A23 & $5.99 \mathrm{def}$ & $3.56 \mathrm{cde}$ & $11.14 b c$ & cream \\
\hline RIL A24 & $6.08 \mathrm{cde}$ & 2.111 & $9.45 \mathrm{fghij}$ & green \\
\hline RIL A25 & $4.51 \mathrm{~m}$ & 3.07 fghij & $10.60 \mathrm{bcd}$ & green \\
\hline RIL A26 & $4.59 \mathrm{Im}$ & 2.81ijk & 6.210 & cream \\
\hline RIL A27 & $6.25 \mathrm{bcd}$ & 3.35def & $11.27 \mathrm{bc}$ & orange \\
\hline RIL A28 & $5.40 \mathrm{ghi}$ & $1.63 \mathrm{~m}$ & 9.99 cdefgh & cream \\
\hline RIL A29 & $4.97 \mathrm{jk}$ & 2.201 & $11.61 \mathrm{~b}$ & orange \\
\hline RIL A30 & $5.90 \mathrm{def}$ & $2.73 \mathrm{jk}$ & $11.14 b c$ & green \\
\hline LSD & 0.38 & 0.38 & 1.04 & ---- \\
\hline
\end{tabular}




\section{2- Genetic Determinations:}

Based on the previous evaluation of ananas inbred lines, 3 testers and 12 inbred lines were selected to make 36 crosses using line $\times$ tester analysis.

\section{a. Heterosis :}

A great attempts were exerted to exploit the heterosis in various traits that lead to high yield to detect the best cross which use as $F_{1}$ hybrid. If Hybrids have great heterosis, they have valuable opportunities to detect favorable lines in consecutive generations as compared to hybrids having less heterotic impacts (Sharif et al., 2001).

The results in Table (4) show that the mean square of replications had nonsignificant differences for all studied traits, whereas, genotypes accounted highly significant for all traits except average fruit weight which has significant differences. The recorded data for crosses were highly significant for all traits. Also, mean square of parents revealed highly significant differences for all traits. Parents vs crosses mean square as an indicator to average heterosis overall crosses were found for most of characters namely LAI, early yield, total yield, marketable yield percentage, average Table 4. The analysis of variance and mean squares for the mating design (Line $x$ Tester analysis) for some melon characters in the open field of 2018 summer season.

\begin{tabular}{|c|c|c|c|c|c|c|c|c|c|c|c|}
\hline $\begin{array}{l}\text { Source of } \\
\text { variance }\end{array}$ & DF & $\begin{array}{l}\text { Leaf } \\
\text { area } \\
\text { index }\end{array}$ & $\begin{array}{c}\text { Early yield } \\
\text { (Ton/feddan) }\end{array}$ & $\begin{array}{l}\text { Total yield } \\
\text { (Ton/feddan) }\end{array}$ & $\begin{array}{c}\text { Marketable } \\
\text { yield } \\
(\%)\end{array}$ & $\begin{array}{l}\text { Average } \\
\text { fruit } \\
\text { weight } \\
(\mathrm{kg}) \\
\end{array}$ & $\begin{array}{l}\text { Fruit } \\
\text { shape } \\
\text { index }\end{array}$ & $\begin{array}{l}\text { Netting } \\
(\%)\end{array}$ & $\begin{array}{c}\text { Seed } \\
\text { cavity } \\
\text { diameter } \\
(\mathrm{cm}) \\
\end{array}$ & $\begin{array}{l}\text { Flesh } \\
\text { thickness } \\
(\mathrm{cm})\end{array}$ & \begin{tabular}{|c|} 
Total \\
Soluble \\
solids \\
$(\%)$ \\
\end{tabular} \\
\hline Replications & 2 & 0.07 & 0.12 & 8.92 & 0.72 & 0.03 & 0.01 & 0.90 & 0.14 & 0.09 & 1.18 \\
\hline Genotypes & 50 & $1.88^{* *}$ & $2.24 * *$ & $41.87 * *$ & $53.95 * *$ & $1.55^{*}$ & $0.43^{* *}$ & $2261.10 * *$ & $2.98^{* *}$ & $2.70^{* *}$ & $14.60 * *$ \\
\hline Crosses $(\mathrm{C})$ & 35 & $1.78^{* *}$ & $2.33^{* *}$ & $41.96 * *$ & $60.58^{* *}$ & $1.65^{* *}$ & $0.46^{* *}$ & $2281.87 * *$ & $3.39 * *$ & $2.89 * *$ & $14.31^{* *}$ \\
\hline Parents(P) & 14 & $1.03^{* *}$ & $1.31 * *$ & $26.74 * *$ & $35.00 * *$ & $0.44 * *$ & $0.34 * *$ & $2344.49 * *$ & $2.14 * *$ & $2.18^{* *}$ & $14.77^{* *}$ \\
\hline Pvs $C$ & 1 & $17.33 * *$ & $12.13^{* *}$ & $250.70^{* *}$ & $86.99 * *$ & $13.84 * *$ & $0.25 *$ & $366.44 * *$ & $0.16^{* *}$ & $3.36^{* *}$ & $22.52 * *$ \\
\hline $\begin{array}{l}\text { Inbred lines } \\
\text { (female) }\end{array}$ & 11 & $1.21^{* *}$ & $1.69 * *$ & $24.62 * *$ & $62.50 * *$ & $1.43^{* *}$ & $0.33^{* *}$ & $2390.00 * *$ & $2.08 * *$ & $1.72^{* *}$ & $11.62 * *$ \\
\hline $\begin{array}{l}\text { Testers } \\
\text { (male) }\end{array}$ & 2 & $13.94 * *$ & $20.30 * *$ & $324.39 * *$ & $42.55^{*}$ & $3.34 * *$ & $5.42^{* *}$ & $12115.15 * *$ & $38.54 * *$ & $18.97 * *$ & $102.15^{* *}$ \\
\hline$L x T$ & 22 & $0.96 * *$ & $1.02 * *$ & $24.95 * *$ & $61.27^{* *}$ & $1.60 * *$ & $0.08^{*}$ & $1333.88^{* *}$ & $0.86^{* *}$ & $2.02^{* *}$ & $7.67 * *$ \\
\hline Error & 100 & 0.08 & 0.11 & 3.75 & 12.16 & 0.05 & 0.04 & 44.63 & 0.13 & 0.12 & 1.11 \\
\hline
\end{tabular}

fruit weight, netting percentage, seed cavity diameter, flesh thickness and TSS. While FSI mean square indicated that the variance due to heterosis illustrating a wide range of heterosis values among the hybrids for most of traits. Inbred lines (female parents) showed high significant for all traits. Testers (male parents) showed high significant for all traits except marketable yield percentage which has significant differences. Line $x$ Tester interaction was highly significant for all the traits except for FSI that has significant differences. Differing for all studied traits in significance that was observed among lines, testers and their $F_{1}$ hybrids for most of the traits which indicated the existence of genetic variances between the genotypes. The significant differences 
resulted between parents and crosses are in agreement with the results reported by Chandhaet al. (2001) and Dhaliwalet al. (2003).

Data in Table (5) show heterosis over mid-parent and better-parent for $36 F_{1}$ hybrids. They revealed significant mid parent heterosis for most of the characters denoting predominance of non-additive gene action in genetic control of these characters.

Highly significant, desirable positive heterosis and the greatest values of heterobeltiosis were observed in the crosses RIL A29 × RIL A5, RIL A30 × RIL A5, RIL A19 $\times$ RIL A5 and RIL A2 × RIL A5 for LAI. Similarly, RIL A3 × RIL A5, RIL A14 × RIL A5, RIL A29 $\times$ RIL A5, RIL A30 $\times$ RIL A5 and RIL A18 $\times$ RIL A20 showed highly significant, desirable positive heterosis and the greatest values of heterobeltiosis for early yield. This result is coincided with Duradundiet al. (2018) who reported that early yield had positive strong heterosis and farmers prefer to grow early and high yielding hybrids in order to catch early market to get higher prices and to avoid

Table 5. Heterosis (\%) values over mid-parent (MP) and better-parent (Heterobeltiosis-BP) of $36 \mathrm{~F}_{1}$ hybrids for some melon characters in the open field of 2018summer season.

\begin{tabular}{|c|c|c|c|c|c|c|c|c|c|c|c|c|}
\hline \multirow{2}{*}{ Crosses } & \multicolumn{2}{|c|}{ Leaf area index } & \multicolumn{2}{|c|}{ Early yield } & \multicolumn{2}{|c|}{ Total vield } & \multicolumn{2}{|c|}{ Marketable } & \multicolumn{2}{|c|}{ Average fruit } & \multicolumn{2}{|c|}{ Fruit } \\
\hline & $\mathrm{MPH}$ & $\mathrm{BPH}$ & $\mathrm{MPH}$ & $\mathrm{BPH}$ & $\mathrm{MPH}$ & $\mathrm{BPH}$ & $\mathrm{MPH}$ & $\mathrm{BPH}$ & $\mathrm{MPH}$ & $\mathrm{BPH}$ & $\mathrm{MPH}$ & $\mathrm{BPH}$ \\
\hline RILA2 $\times$ RILA5 & $67.97 * *$ & $25,70^{* *}$ & $49.55 * *$ & $11.72^{*}$ & 4.59 & -12.29 & 1.35 & -2.49 & $36.55 * *$ & $21.92 * *$ & -3.80 & -13.03 \\
\hline RIL A3 x RIL A5 & $34.54 * *$ & \begin{tabular}{|l|}
$10.70^{*}$ \\
\end{tabular} & $143.47 * *$ & 69.33** & $21.85^{* *}$ & 5.84 & -8.53 & -10.78 & $30.57 * *$ & $18.92 * *$ & -12.07 & -22.59 \\
\hline RILA7x RILA5 & $44.15 * *$ & \begin{tabular}{|l|}
3.89 \\
\end{tabular} & $28.46 * *$ & -3.94 & $17.61 * *$ & -4.11 & 1.72 & -1.00 & $49.77^{* *}$ & $21.04 * *$ & -22.78 & -17.04 \\
\hline RIL A8 x RIL A5 & $51.98 * *$ & $22.04 * *$ & -3.74 & -34.77 & $28.24 * *$ & $8.14 *$ & -7.07 & -10.35 & $47.76 * *$ & $23.33^{* *}$ & -15.24 & -17.62 \\
\hline RILA14 x RILA5 & $62.46 * *$ & $19.65 * *$ & $121.89 * *$ & $51.41 * *$ & $13.57^{*}$ & -4.90 & -0.78 & -5.66 & $47,49 * *$ & $44.18^{* *}$ & -7.37 & -0.99 \\
\hline RIL A18 x RIL A5 & $45.76^{* *}$ & $18.01^{* *}$ & $59.65 * *$ & $8.18^{*}$ & $31.85 * *$ & 2.64 & -0.42 & -12.36 & $84.77 * *$ & $65.92 * *$ & -21.78 & -32.91 \\
\hline RIL A19 x RIL A5 & $61.72^{* *}$ & $29.23 * *$ & $31.45 * *$ & 6.66 & 6.03 & -10.47 & 1.44 & -2.39 & $55.44 * *$ & $34.82 * *$ & -19.81 & -11.69 \\
\hline RIL A22 x RIL A5 & $41.83 * *$ & $14.73 * *$ & $61.22 * *$ & $8.11^{*}$ & $31.94 * *$ & 1.55 & -4.19 & -11.35 & $66.85^{* *}$ & $54.08^{* *}$ & -35.72 & -36.35 \\
\hline RIL A23 x RIL A5 & $52.54 * *$ & $8.43^{*}$ & $72.05^{* *}$ & $27.14 * *$ & $23.57 * *$ & -3.32 & -0.48 & -3.43 & $78.15^{* *}$ & $38.70^{* *}$ & -18.30 & -15.13 \\
\hline RIL A26 x RIL A5 & $43.62 * *$ & $21.29 * *$ & $91.87 * *$ & $23.82 * *$ & $14.56^{*}$ & -17.01 & -7.37 & -8.69 & $51.31 * *$ & $43.12 * *$ & -29.23 & -12.46 \\
\hline RIL A29 x RIL A5 & $64.73 * *$ & $38.69 * *$ & $97.69 * *$ & $35.47 * *$ & $9.03^{*}$ & -12.13 & -3.92 & -5.42 & $41.11^{* *}$ & $23.69 * *$ & 1.01 & $8.95 *$ \\
\hline RIL $A 30 \times R$ & $77.27 * *$ & $29.86 * *$ & $78.19 * *$ & $31.79 * *$ & $39.65 * *$ & $13.61 *$ & -0.41 & -3.29 & $75.00 * *$ & $51.07 * *$ & -24.65 & -29.28 \\
\hline RIL $A 2 \times R$ & $16.64 *$ & -13.78 & $34.63 * *$ & -2.02 & -16.08 & -25.99 & -2.75 & -5.22 & 0.09 & -13.25 & $20.49 * *$ & $8.32 *$ \\
\hline RIL A3 $\times$ R & -17.48 & -33.09 & $33.77^{* *}$ & 5.41 & -15.00 & -22.15 & -4.44 & -5.56 & 3.24 & -8.80 & $21.13^{* *}$ & 6.05 \\
\hline RILA7x RILA10 & -24.16 & -45.96 & $51.33 * *$ & $10.05^{*}$ & -15.08 & -27.33 & -2.11 & -3.48 & -28.81 & -43.93 & $9.47 *$ & $16.81 * *$ \\
\hline RIL A8 x RIL A10 & $13.64 *$ & -10.01 & $39.29 * *$ & $13.77^{*}$ & $13.60^{*}$ & 0.79 & -2.58 & -14.57 & $91.23 * *$ & $55.35 * *$ & $25.03 * *$ & $20.77 * *$ \\
\hline RIL A14 x RIL A10 & 9.46 & -20.34 & $43.28 * *$ & $15.90 *$ & -27.19 & -35.88 & -4.27 & -7.80 & -17.17 & -21.67 & $19.62 * *$ & $27.00 * *$ \\
\hline RIL A18 x RIL A10 & -25.15 & -40.25 & $13.03 *$ & -7.69 & $11.16^{*}$ & -9.50 & 1.17 & 0.28 & -20.90 & -31.07 & $13.91^{* *}$ & -2.82 \\
\hline RIL A19 x RIL A10 & -21.10 & -37.82 & $15.03 *$ & -21.71 & -24.71 & -33.10 & -5.09 & -7.48 & -28.00 & -39.31 & $10.78^{*}$ & $21.15^{* *}$ \\
\hline RIL A22 x RIL A10 & -10.17 & -28.35 & $36.76 * *$ & $13.49 *$ & $35.59 * *$ & $9.04 *$ & 0.64 & -5.72 & $77.77 * *$ & $59.14 * *$ & $18.51 * *$ & $16.62 * *$ \\
\hline RIL A23 x RIL A10 & $37.15^{* *}$ & -3.58 & -8.47 & -32.67 & \begin{tabular}{|l|}
-1.68 \\
\end{tabular} & -19.52 & -1.63 & -3.29 & $62.53 * *$ & $23.52 * *$ & $21.79 * *$ & $25.68^{* *}$ \\
\hline RIL $A 26 x$ & -11.82 & -26.65 & $29.24 * *$ & $14.70^{*}$ & -4.80 & -28.30 & -2.02 & -2.13 & $60.56 * *$ & $47,10 * *$ & $19.13 * *$ & $46.25 * *$ \\
\hline RIL A29 x R & -21.22 & -34.67 & -18.53 & -34.53 & -27.67 & -38.88 & -3.03 & -3.27 & -24.46 & -35.68 & $39.16^{* *}$ & $49.08 * *$ \\
\hline RIL A30 x RIL A10 & -22.84 & -44.14 & 7.77 & -20.78 & -7.51 & -21.04 & -9.28 & -10.75 & 2.21 & -14.24 & $21.36 * *$ & $13.22 * *$ \\
\hline RIL A2 $\times$ RILA20 & $65.07 * *$ & $19.06 * *$ & $26.40 * *$ & $21,30 * *$ & $29.94 * *$ & $17.73 * *$ & -6.46 & -6.11 & $53,56 * *$ & $20.57 * *$ & $12.76 * *$ & 4.11 \\
\hline RIL A3 x RIL A20 & $12.09 *$ & -11.72 & 3.63 & -10.50 & -20.70 & -25.30 & -4.98 & -3.24 & -16.43 & -33.30 & -7.52 & -16.91 \\
\hline RIL A7x RIL A20 & -17.77 & -42.72 & $25.91 * *$ & $20.99 * *$ & $21.37 * *$ & 6.59 & 2.97 & $4.60^{*}$ & -26.45 & -46.77 & -24.58 & -16.91 \\
\hline RIL $A 8 \times$ RIL A20 & -26.04 & -43.06 & $18.79 * *$ & -1.35 & -12.21 & -19.96 & 2.72 & 3.39 & -24.56 & -43.95 & -20.87 & -21.32 \\
\hline RIL A14 x RIL A20 & $56.93 * *$ & $11.53^{*}$ & $20.05^{* *}$ & 0.74 & -21.27 & -28.78 & $7.51^{*}$ & $6.60 * *$ & $55.28 * *$ & $31.47^{* *}$ & -16.88 & -8.91 \\
\hline RIL $A 18 \times R$ & 6.99 & -17.00 & $63.26 * *$ & $35.59 * *$ & -16.28 & -30.17 & 0.26 & 2.41 & $32.84 * *$ & 4.79 & -22.18 & -31.93 \\
\hline RIL $A 19 \times R$ & -36.55 & -51.37 & -10.47 & -3.37 & $29.67 * *$ & $18.40 * *$ & -3.78 & -3.39 & -19.36 & -38.18 & -8.74 & 3.11 \\
\hline RIL A22 $\times$ R & $-49,29$ & -60.69 & -6.14 & -23.28 & -8.35 & -24.53 & -8.51 & -11.84 & -43.60 & -54.47 & $-4,20$ & -2.90 \\
\hline RIL A23 x R & $72.28 * *$ & $18.49 * *$ & $21.04 * *$ & $14.33^{*}$ & $37.67 * *$ & $15.47 * *$ & -2.68 & -1.43 & $97.27 * *$ & $38.53 * *$ & -32.47 & -28.13 \\
\hline RILA26 x RIL A20 & -33.61 & -46.42 & -12.40 & -32.52 & -15.26 & -34.82 & -1.03 & -8.39 & -39.27 & -49.98 & -21.77 & -0.50 \\
\hline RIL A29 x RIL A20 & -40.67 & -52.27 & -0.27 & -15.75 & 1.41 & -12.09 & 4.55 & $7.49 * *$ & -27.35 & -43.82 & -13.66 & -4.50 \\
\hline RILA30 x RILA20 & $57.24 * *$ & $11.19 *$ & $15.54 *$ & $9.27^{*}$ & $29.00 * *$ & $13.01 *$ & $5.28 *$ & $6.71 * *$ & $55,36 * *$ & $18.65 * *$ & -14.86 & $-18,32$ \\
\hline LSD $1 \%$ & 0.46 & 0.53 & 0.55 & 0.63 & 3.23 & 3.73 & 5.82 & 6.72 & 0.36 & 0.41 & 0.33 & 0.38 \\
\hline LSD $5 \%$ & 0.32 & 0.37 & 0.39 & 0.45 & 2.27 & 2.63 & 4.09 & 4.73 & 0.25 & 0.29 & 0.23 & 0.27 \\
\hline
\end{tabular}


market glut therefore earliness is an important trait in vegetables like muskmelon. Regarding the total yield, the crosses RIL A19 $\times$ RIL A20, RIL A2 $\times$ RIL A20, RIL A23 $\times$ RIL A20, RIL A30 × RIL A20, RIL A22 × RIL A10, RIL A30 × RIL A5 and RIL A8 $\times$ RIL A5 showed highly significant, desirable positive heterosis and the greatest values of heterobeltiosis for this trait. With respect to marketable yield percentage, the crosses RIL A14 $\times$ RIL A20 and RIL A30 × RIL A20 had significant and positive desirable heterosis. Also, the cross RIL A29 $\times$ RIL A20 had highly significant and positive desirable heterobeltiosis, while RIL A7 $\times$ RIL A20 had significant heterobeltiosis for this trait. Likewise, highly significant, desirable positive heterosis and the greatest values of heterobeltiosis were observed in the crosses RIL A18 $\times$ RIL A5, RIL A22 × RIL A10, RIL A8 × RIL A10, RIL A22 × RIL A5 and RIL A30 × RIL A5 for average fruit weight. In the same trend, crosses RIL A29 $\times$ RIL A10, RIL A23 $\times$ RIL A10 and RIL A8 $\times$ RIL A10 showed positive and highly significant heterosis and heterobeltiosis for FSI. The crosses RIL A30 × RIL A10, RIL A3 $\times$ RIL A10 and RIL A2 $\times$ RIL A10 showed highly significant and great values of positive heterosis, while in RIL A26 × RIL A10, RIL A14 $\times$ RIL A10 and RIL A19 $\times$ RIL A10 showed highly significant and great values of positive heterobeltiosis for FSI. These results are in agreement with Riggs (1988) who reported that the main aim of any breeding program is to enhance the yielding ability of the crop. Heterosis breeding offers quick and quantum jump in yield. $F_{1}$ hybrids derived from crossing of pure lines are exceptionally uniform in growth and development as well as possess better quality and adaptability to varied environmental conditions and give high early and total yields and can be exploited in rapid deployment of dominant genes for resistance to diseases and pests. Also, Duradundiet al. (2018) reported that higher magnitude of heterosis was observed for the yield components and average fruit weight.

Data in Table (6) show heterosis over mid-parent (MP) and better-parent (Heterobeltiosis-BP) of $36 \mathrm{~F}_{1}$ hybrids for the rest four traits.

Netting percentage showed highly significant heterosis in crosses RIL A2 $\times$ RIL A5, RIL A3 × RIL A5, RIL A18× RIL A5, RIL A19 × RIL A5, RIL A23 × RIL A5, RIL A26 $\times$ RIL A5, RIL A29 × RIL A5, RIL A3 × RIL A20, RIL A18 × RIL A20 and RIL A26 × RIL $A 20$, while non-significant heterobeltiosis was shown in all crosses for the same trait. Concerning seed cavity diameter, the crosses RIL A23 $\times$ RIL A10, RIL A18 $\times$ RIL A10, RIL A8× RIL A10, RIL A29 × RIL A10, RIL A19 × RIL A5 and RIL A7 × RIL A5 showed positive highly significant heterosis and the greatest values of heterobeltiosis for this trait. Referring the flesh thickness, the crosses RIL A29 $\times$ RIL A5, RIL A14 $\times$ RIL A5, RIL A19 $\times$ RIL A5 and RIL A8 $\times$ RIL A5 showed desirable highly significant 
heterosis and significant heterobeltiosis. Also, the crosses RIL A2 $\times$ RIL A5, RIL A23 $\times$ RIL A5, RIL A30 × RIL A5, RIL A14 × RIL A20, RIL A19 × RIL A20 and RIL A29 × RIL Table 6. Heterosis (\%) values over mid-parent (MP) and better-parent (Heterobeltiosis-BP) of $36 F_{1}$ hybrids for some

\begin{tabular}{|c|c|c|c|c|c|c|c|c|}
\hline \multirow[t]{2}{*}{ Crosses } & \multicolumn{2}{|c|}{$\begin{array}{l}\text { Netting } \\
(\%)\end{array}$} & \multicolumn{2}{|c|}{$\begin{array}{l}\text { Seed cavity } \\
\text { diameter } \\
(\mathrm{cm})\end{array}$} & \multicolumn{2}{|c|}{$\begin{array}{l}\text { Flesh thickness } \\
\qquad(\mathrm{cm})\end{array}$} & \multicolumn{2}{|c|}{$\begin{array}{c}\text { Total soluble } \\
\text { solids } \\
(\%)\end{array}$} \\
\hline & $\begin{array}{l}\text { MPH } \\
(\%)\end{array}$ & $\begin{array}{l}\text { BPH } \\
(\%)\end{array}$ & $\begin{array}{l}\text { MPH } \\
(\%)\end{array}$ & $\begin{array}{l}\mathrm{BPH} \\
(\%) \\
\end{array}$ & $\begin{array}{l}\text { MPH } \\
(\%)\end{array}$ & $\begin{array}{l}\text { BPH } \\
(\%)\end{array}$ & $\begin{array}{l}\text { MPH } \\
(\%)\end{array}$ & $\begin{array}{l}\text { BPH } \\
(\%)\end{array}$ \\
\hline RIL A2 $\times$ RIL A5 & $21.62^{* *}$ & 0.00 & 7.85 & $39.64 * *$ & $19.53^{* *}$ & 3.56 & $21.13^{* *}$ & 2.74 \\
\hline RIL A3 x RIL A5 & $15.51 * *$ & 0.00 & $23.52 * *$ & $33.15 * *$ & $11.46^{*}$ & -7.26 & $22.49 * *$ & $6.45 *$ \\
\hline RIL A7x RIL A5 & 4.95 & 0.00 & $32.24 * *$ & $57.13 * *$ & $8.27 *$ & 0.00 & $22.82 * *$ & $6.11 *$ \\
\hline RIL A8 x RIL A5 & 0.00 & 0.00 & $21.42 * *$ & $30.42 * *$ & $22.27 * *$ & $7.33 *$ & 5.03 & -2.49 \\
\hline RIL A14 x RIL A5 & 0.00 & 0.00 & $9.02 *$ & $24.56 * *$ & $29.97 * *$ & $5.91 *$ & $15.04 * *$ & $4.20 *$ \\
\hline RIL A18 x RIL A5 & $100.00 * *$ & 0.00 & -1.05 & 6.21 & 6.68 & 0.57 & 8.64 & -9.85 \\
\hline RIL A19 x RIL A5 & $34.44 * *$ & 0.00 & $26.69 * *$ & $57.28 * *$ & $29.70 * *$ & $9.25 *$ & $12.29 *$ & 1.42 \\
\hline RIL A22 x RIL A5 & $7.91 *$ & 0.00 & $27.94 * *$ & $30.03 * *$ & $7.39 *$ & 1.78 & $17.68 * *$ & $3.97 *$ \\
\hline RIL A23 x RIL A5 & $27.00 * *$ & 0.00 & $17.98 * *$ & $38.62 * *$ & $23.30 * *$ & 0.71 & $10.83^{* *}$ & $3.32 *$ \\
\hline RIL A26 x RIL A5 & $20.35 * *$ & 0.00 & $26.25 * *$ & $28.31^{* *}$ & $12.08 *$ & -7.47 & $23.10 * *$ & -8.80 \\
\hline RIL A29 x RIL A5 & $20.35 * *$ & 0.00 & $22.66 * *$ & $30.03 * *$ & $40.00 * *$ & $5.70 *$ & 6.89 & 1.58 \\
\hline RIL A30 x RIL A5 & 0.00 & 0.00 & $22.04 * *$ & $42.13 * *$ & $24.17 * *$ & 1.43 & $15.67 * *$ & $7.80 *$ \\
\hline RIL A2 x RIL A10 & -8.78 & -25.00 & -1.71 & $36.93 * *$ & 5.27 & -9.73 & -1.03 & -18.15 \\
\hline RIL A3 x RIL A10 & -5.28 & -18.00 & $24.07 * *$ & $42.63 * *$ & -28.03 & -40.70 & -17.21 & -29.91 \\
\hline RIL A7x RIL A10 & -33.53 & -36.67 & $21.65 * *$ & $54.92 * *$ & -28.91 & -35.08 & -17.89 & -30.89 \\
\hline RIL A8 x RIL A10 & 0.00 & 0.00 & $39.87 * *$ & $60.19 * *$ & -0.48 & -13.55 & $10.43 *$ & -0.36 \\
\hline RIL A14 x RIL A10 & 0.00 & 0.00 & 1.47 & $24.02 * *$ & -7.96 & -25.70 & -16.36 & -26.31 \\
\hline RIL A18 x RIL A10 & -100.00 & -100.00 & $39.97 * *$ & $60.19 * *$ & 3.57 & -3.48 & -9.80 & -26.96 \\
\hline RIL A19 x RIL A10 & -26.06 & -45.00 & $11.25^{*}$ & $48.34 * *$ & -37.97 & -48.27 & -19.35 & -29.14 \\
\hline RIL A22 x RIL A10 & -44.25 & -48.33 & -4.54 & 3.13 & -5.87 & -11.82 & $11.53 *$ & -4.07 \\
\hline RIL A23 x RIL A10 & -61.90 & -70.00 & $45.76 * *$ & $83.45 * *$ & -26.62 & -40.63 & -25.87 & -32.85 \\
\hline RIL A26 x RIL A10 & -27.79 & -40.00 & $8.46^{*}$ & $17.18^{* *}$ & -46.50 & -56.26 & -20.74 & -42.44 \\
\hline RIL A29 x RIL A10 & -41.83 & -51.67 & $41.05 * *$ & $59.31 * *$ & -5.41 & -29.18 & -19.72 & -25.92 \\
\hline RIL A30 x RIL A10 & 0.00 & 0.00 & $21.07 * *$ & $50.97 * *$ & -40.35 & -51.74 & -18.75 & -26.42 \\
\hline RIL A2 x RIL A20 & -19.73 & -34.00 & -35.83 & -17.69 & -33.85 & -46.04 & $17.68 * *$ & -2.42 \\
\hline RIL A3 x RIL A20 & $15.51^{* *}$ & 0.00 & -33.45 & -28.84 & -35.10 & -48.98 & -5.75 & -19.98 \\
\hline RIL A7x RIL A20 & -43.68 & -46.33 & -20.65 & -6.54 & -6.36 & -19.00 & -6.50 & -21.08 \\
\hline RIL A8 x RIL A20 & 0.00 & 0.00 & -10.62 & -4.77 & -41.69 & -51.87 & -24.32 & -31.51 \\
\hline RIL A14 x RIL A20 & 0.00 & 0.00 & -23.75 & -13.61 & $20.84 * *$ & -6.80 & -2.49 & -13.83 \\
\hline RIL A18 x RIL A20 & $60.00 * *$ & -20.00 & -11.36 & -5.61 & -52.35 & -58.00 & -17.80 & -33.26 \\
\hline RIL A19 x RIL A20 & -64.15 & -73.33 & -18.41 & 0.39 & $16.86^{* *}$ & -7.11 & $12.61 *$ & -0.77 \\
\hline RIL A22 x RIL A20 & $7.91 *$ & 0.00 & -29.83 & -29.23 & 5.64 & -6.43 & -20.34 & -31.29 \\
\hline RIL A23 x RIL A20 & -21.68 & -38.33 & -17.45 & -3.84 & -23.68 & -41.01 & -3.84 & -12.63 \\
\hline RIL A26 x RIL A20 & $20.35^{* *}$ & 0.00 & -18.01 & -17.30 & -18.29 & -36.23 & -35.74 & -53.24 \\
\hline RIL A29 x RIL A20 & -59.08 & -66.00 & -15.15 & -10.77 & $20.99 * *$ & -12.93 & -12.80 & -19.28 \\
\hline RIL A30 x RIL A20 & 0.00 & 0.00 & -9.40 & 4.62 & 6.30 & -17.84 & -27.02 & -33.70 \\
\hline LSD $1 \%$ & 11.15 & 12.87 & 0.61 & 0.70 & 0.59 & 0.68 & 1.76 & 2.03 \\
\hline LSD 5\% & 7.84 & 9.06 & 0.43 & 0.49 & 0.41 & 0.48 & 1.24 & 1.43 \\
\hline
\end{tabular}

A20 had desirable highly significant heterosis, while the crosses RIL A3 $\times$ RIL A5,RIL A7 $\times$ RIL A5, RIL A22 $\times$ RIL A5 and RIL A26 $\times$ RIL A5 had desirable significant heterosis for this trait. Regarding TSS, the crosses RIL A30 $\times$ RIL A5, RIL A3 $\times$ RIL A5, RIL A7 $\times$ RIL A5, RIL A14 $\times$ RIL A5, RIL A22 × RIL A5 and RIL A23 × RIL A5 showed desirable highly significant heterosis and significant heterobeltiosis. Also, the 
crosses RIL A26 $\times$ RIL A5, RIL A2 $\times$ RIL A5 and RIL A2 $\times$ RIL A20 had desirable highly significant heterosis, while the crosses RIL A19 × RIL A20, RIL A19 × RIL A5, RIL A2 $\times$ RIL A10 and RIL A8 $\times$ RIL A10 had desirable significant heterosis for this trait. The fundamental target of breeding is to get heterosis for yield that correlated with heterosis for other characters. However, yield is a complex character where crosses may be considered for further study of combining ability.

The detected significant heterosis over better parent in most of the hybrids for whole characters denoted the presence of non-additive gene action in genetic control of those characters. Supposing that epistasis is disappeared, the reason of heterosis may only be due to the dominant gene action. This result was coincided with former results of Sharma et al. (2006).

\section{b. Combining Ability :}

Determination of general combining ability (GCA) supplies fundamental and essential data for utilizing genetic vigor of parents for developing the best and top lines or hybrids. The significant and great GCA impacts of a parent line indicating the existence of preferable additive genes with additive inheritance impacts which lead to select in recent generations for improving greatly adapted hybrids(Roy et al.,2002).

Data in Table (7) show the estimated values for general combining ability effects. General combining ability studies the estimates of variation due to GCA is portioned for both inbred lines (females) and testers (males) parents for most of traits to discover the potential parents for further breeding and selecting programs. In case of inbred line T5 showed positive highly significant GCA effects for all characters except early yield and FSI, but it had positive significant GCA effect for marketable yield percentage. Meanwhile, positive highly significant GCA effects of the most inbred lines and testers were shown for whole characters. The inbred line T5 showed the ultimate positive GCA impacts of the most characters, so this parent could be strongly utilized in future breeding programs. However, inbred lines T10 and T20 showed highly significant negative GCA effects of the most traits. So, the inbred line (male) T5, which had the highly significant positive GCA effects, is the potential parent (good combiner) that could be used in selection program and would be effective for its efficient use in subsequent crossing for development the yield and the most of fruit quality. Although the inbred line T10 had negative GCA effects of the most traits, it showed positive highly significant GCA effects of early yield, average fruit weight, FSI and seed cavity diameter. So, the inbred line T10 is considered as potential parent for earliness, which is very important trait for melon's farmers. 
The RIL A14 (Female) had highly significant positive GCA effects (good combiner) for LAI, early yield, average fruit weight, netting percentage and flesh thickness, but it had significant positive GCA effects for FSI. Also, the RIL A22

Table 7. Estimation of parental general combining ability effects (GCA) for some melon characters in the open field of 2018 summer season.

\begin{tabular}{|c|c|c|c|c|c|c|c|c|c|c|}
\hline Genotypes & $\begin{array}{l}\text { Leaf } \\
\text { area } \\
\text { index } \\
\end{array}$ & $\begin{array}{l}\text { Early yield } \\
\text { (Ton/feddan } \\
\text { ) }\end{array}$ & $\begin{array}{c}\text { Total yield } \\
\text { (Ton/feddan } \\
\text { ) }\end{array}$ & $\begin{array}{c}\text { Marketable } \\
\text { yield } \\
(\%)\end{array}$ & $\begin{array}{l}\text { Average } \\
\text { fruit } \\
\text { weight }\end{array}$ & $\begin{array}{l}\text { Fruit } \\
\text { shape } \\
\text { index }\end{array}$ & $\begin{array}{l}\text { Netting } \\
(\%)\end{array}$ & $\begin{array}{l}\text { Seed } \\
\text { Cavity } \\
\text { diameter }\end{array}$ & \begin{tabular}{|c|} 
Flesh \\
thickness \\
$(\mathrm{cm})$
\end{tabular} \mid & $\begin{array}{l}\text { Total } \\
\text { soluble } \\
\text { solids }\end{array}$ \\
\hline \multicolumn{11}{|c|}{ Inbred lines(Females) } \\
\hline RII A2 & $588 * *$ & -0.176 & 0.561 & -0.290 & $0.150 *$ & 0.037 & 0.130 & -0.130 & 0.058 & $1.415 * *$ \\
\hline $\mathrm{RIL} A 3$ & -0.026 & $0.426 * *$ & -0.442 & & -0.3 & $-0.148 *$ & $13.796 * *$ & $-0.311 * *$ & $-0.660 * *$ & 0.105 \\
\hline RIL A7 & & -0.121 & 0.400 & & -0.6 & -0.053 & $-7.870 * *$ & $0.508 * *$ & 0.087 & -0.017 \\
\hline $\mathrm{RIL}$ A8 & -0.025 & & $1.452 *$ & -2.9 & & -0.057 & $19.796 * *$ & $0.220 *$ & -0.050 & $0.600 *$ \\
\hline $\mathrm{RIL} \mathrm{A} 14$ & $0.400 * *$ & 0.63 & -2.24 & & 0.32 & $0.145 *$ & $19.796 * *$ & $-0.449 * *$ & $0.550 * *$ & 0.492 \\
\hline RILA18 & -0.079 & 0.33 & & & & $=0.344 * *$ & $-0.204 * *$ & -0.136 & -0.106 & $-1.197 * *$ \\
\hline RILA19 & $-0.298 * *$ & -0.80 & & & & $0.115^{*}$ & $-9.648 * *$ & $0.527 * *$ & $0.235 *$ & $0.875 * *$ \\
\hline RII A22 & $-0.431 * *$ & & & -4.9 & & -0.088 & 3.685 & $-0.861 * *$ & $0.714 * *$ & $0.731 *$ \\
\hline RIL A23 & $0.523 * *$ & -0.4 & & & 0.65 & -0.051 & $-6.315 * *$ & $0.645 * *$ & $-0.390 * *$ & 0.178 \\
\hline RIL A26 & $-0.217 * *$ & 0.52 & $-2.977 * *$ & & 0 & $0.239 * *$ & $6.463 * *$ & $-0.535 * *$ & $-0.681 * *$ & $-2.947 * *$ \\
\hline RII A29 & $-0.195 *$ & -0.23 & & $6 * *$ & $-0.547 * *$ & $0.357 * *$ & $-9.426 * *$ & 0.117 & $0.380 * *$ & 0.111 \\
\hline RIL $A 30$ & $0.269 * *$ & $-0.315 * *$ & $2.403 * *$ & 503 & $0.310 * *$ & $-0.153 *$ & $19.796 * *$ & $0.406 * *$ & -0.137 & -0.347 \\
\hline ISD $5 \%$ & 0.152 & & 1.0 & 1.92 & 0.119 & 0.109 & 3.697 & 0.201 & 0.194 & 0.583 \\
\hline ISD $1 \%$ & 0.216 & 0.259 & 1.524 & 2.743 & 0.169 & 0.155 & 5.255 & 0.286 & 0.276 & 0.829 \\
\hline \multicolumn{11}{|c|}{ Testers (Males) } \\
\hline T5 & $0.691 * *$ & $-0.209 * *$ & & $1.245 *$ & $0.334 * *$ & $-0.231 * *$ & $19.796 * *$ & $0.715 * *$ & & $1.942 * *$ \\
\hline T10 & $-0.516 * *$ & 0.833 & -2.3 & -0.48 & 0.26 & $0.448 * *$ & $-6.426 * *$ & $0.472 * *$ & $-0.645 * *$ & $-1.061 * *$ \\
\hline $\mathrm{T} 20$ & $-0.174 * *$ & -0.62 & $56 * *$ & -0.7 & & $-0.217 * *$ & $-3.370 * *$ & $-1.186 * *$ & -0.1 & -0.8 \\
\hline LSD $5 \%$ & 0.076 & & & 0.9 & & & 1.848 & 0.100 & 0.097 & 0.291 \\
\hline$\triangle \mathrm{SD} 1 \%$ & 0.108 & 0.129 & 0.762 & 1.371 & 0.084 & 0.077 & 2.628 & 0.143 & 0.138 & 0.414 \\
\hline
\end{tabular}

(Female) showed significant positive GCA effects for early yield, total yield and TSS, but it produced highly significant positive GCA effects for average fruit weight and flesh thickness. Besides, the RIL A30(Female) exhibited highly significant positive GCA effects for LAI, total yield, average fruit weight, netting percentage and seed cavity diameter, but it had significant positive GCA effects for FSI. Generally, the LAI, early yield, total yield and average fruit weight are very important traits that contribute to great yield and fruit quality.

The inbred line that recorded highly significant negative GCA effects was RIL A3 for average fruit weight, seed cavity diameter and flesh thickness, while RIL A7 for LAI, average fruit weight and netting percentage and RIL A8 for marketable yield. Also, the inbred lines that recorded significant negative GCA effects were RIL A3 for marketable yield and FSI; RIL A26 for marketable yield; RIL A29 for LAI and early yield; RIL A30 for FSI as well as T20 for average fruit weight. So, the inbred lines, which had negative GCA effects, are poor combiners for these traits that make depression of these traits in their $F_{1}$ hybrids.

Accrual of additive gene impacts for preferable traits is a fundamental necessity for genetic improvement and hybrids with great SCA impacts of different characters 
including either one or both of the parents with positive GCA denoting the superiority of additive genetic impacts. In contrast, hybrids with significant and positive SCA including the parents with little or non-significant GCA exhibited the valuable of nonadditive genetic impacts. Several hybrids have high significant SCA impacts in high $\mathrm{x}$ low or high $\mathrm{x}$ high general combining ability combinations refer to the interaction of dominant alleles from good combiners and recessive alleles from poor combiner (Roy et al., 2002). The SCA impacts are accompanied by dominance and epistatic components of variation i.e. fundamentally non-fixable components of variation. Significant specific combining ability is the explanation of relative value of interactions in measuring the behavior of single crosses. Yield and its components per plant is an ultimate objective of melon breeding and genetic development programs.

Data in Table (8) show the estimated values for specific combining ability effects (SCA). The cross RIL A2 $\times$ RIL A5 showed highly significant positive specific combining ability effects for marketable yield, but it had highly significant negative specific combining ability effects for LAI, total yield and average fruit weight, and significant negative specific combining ability effects for early yield and TSS. In the same trend, the cross RIL A19 $\times$ RIL A5 exhibited highly significant positive specific combining ability effects for LAI, average fruit weight and netting percentage and significant positive specific combining ability effects for marketable yield and seed cavity diameter, but it had highly significant negative specific combining ability effects for total yield. Also, the cross RIL A8 $\times$ RIL A10 included highly significant positive specific combining ability effects for LAI, early yield, average fruit weight, netting percentage, flesh thickness and TSS, and significant positive specific combining ability effects for total yield and seed cavity diameter, but it had highly significant negative specific combining ability effects for marketable yield only. As for the cross RIL A22 $\times$ RIL A10 showed highly significant positive specific combining ability effects for LAI, total yield, marketable yield, average fruit weight and TSS, and significant positive specific combining ability effects for early yield only, but it had highly significant negative specific combining ability effects for netting percentage and seed cavity diameter.

Likewise, the cross RIL A2 $\times$ RIL A20 showed highly significant positive specific combining ability effects for LAI, total yield, average fruit weight and TSS; significant positive specific combining ability effects for FSI only, while it had highly significant negative specific combining ability effects for marketable yield, netting percentage and flesh thickness. The cross RIL A14 $\times$ RIL A20 showed highly significant positive specific combining ability effects for LAI, average fruit weight and flesh thickness, besides significant positive specific combining ability effects for marketable yield only, 
but it had significant negative specific combining ability effects for early yield only. As for the cross RIL A18 $\times$ RIL A20 showed highly significant positive specific combining ability effects for early yield, average fruit weight and netting percentage; significant Table 8. Estimation of specific combining ability (SCA) effects for some melon characters in the open field 2018 summer season.

\begin{tabular}{|c|c|c|c|c|c|c|c|c|c|c|}
\hline Crosses & $\begin{array}{c}\text { Leaf area } \\
\text { index }\end{array}$ & $\begin{array}{c}\text { Early yield } \\
\text { (Ton/feddan) }\end{array}$ & $\begin{array}{c}\text { Total yield } \\
\text { (Ton/feddan) }\end{array}$ & $\begin{array}{c}\text { Marketable } \\
\text { yield } \\
(\%)\end{array}$ & $\begin{array}{l}\text { Average } \\
\text { fruit } \\
\text { weight } \\
\text { (kg) }\end{array}$ & $\begin{array}{c}\text { Fruit } \\
\text { shape } \\
\text { index }\end{array}$ & $\begin{array}{l}\text { Netting } \\
(\%)\end{array}$ & $\begin{array}{l}\text { Seed } \\
\text { Cavity } \\
\text { diameter } \\
(\mathrm{cm})\end{array}$ & \begin{tabular}{|c|} 
Flesh \\
thickness \\
$(\mathrm{cm})$
\end{tabular} & $\begin{array}{c}\text { Total } \\
\text { soluble } \\
\text { solids } \\
(\%)\end{array}$ \\
\hline RIL A2 x RIL A5 & $-0.442 * *$ & $-0.406^{*}$ & $-2.573 *$ & $4.086 *$ & $-0.430 * *$ & 0.026 & -0.130 & 0.336 & 0.025 & $-1.219 *$ \\
\hline RIL A3 x RIL A5 & -0.225 & $0.665^{* *}$ & $2.234 *$ & -1.973 & -0.031 & 0.045 & $-13.796 * *$ & 0.241 & 0.236 & 0.624 \\
\hline RIL A7x RIL A5 & 0.079 & $-0.721 * *$ & -0.695 & 1.344 & $0.349 * *$ & 0.046 & $7.870^{*}$ & $0.445^{*}$ & -0.170 & 0.697 \\
\hline RIL A8 x RIL A5 & 0.074 & $-0.911 * *$ & 0.823 & -0.834 & $-0.315^{* *}$ & 0.041 & $-19.796 * *$ & $-0.407^{*}$ & 0.310 & $-1.153^{*}$ \\
\hline RIL A14 x RIL A5 & $-0.414 * *$ & $0.316^{*}$ & 1.786 & -0.883 & -0.188 & 0.128 & $-19.796 * *$ & 0.012 & $-0.357 *$ & -0.086 \\
\hline RIL A18 x RIL A5 & 0.021 & -0.283 & 1.287 & $-6.483 * *$ & $0.380^{* *}$ & 0.061 & $20.204 * *$ & $-1.084 *$ & 0.048 & -0.413 \\
\hline RIL A19 x RIL A5 & $0.537^{* *}$ & -0.223 & $-1.915^{* *}$ & $4.060 *$ & $0.417^{* *}$ & -0.028 & $19.648^{* *}$ & $0.433^{*}$ & 0.114 & -0.869 \\
\hline RIL A22 x RIL A5 & $0.287^{*}$ & -0.047 & -0.344 & 0.244 & 0.137 & $-0.255^{*}$ & -3.685 & $0.658^{* *}$ & $-0.715^{* *}$ & -0.358 \\
\hline RIL A23 x RIL A5 & $-0.834 * *$ & 0.193 & -1.586 & 1.505 & $-0.622 * *$ & 0.078 & $16.315^{* *}$ & $-0.481 *$ & $0.340 *$ & 0.102 \\
\hline RIL A26 x RIL A5 & 0.246 & $0.507^{* *}$ & -0.025 & -0.186 & 0.041 & -0.165 & \begin{tabular}{|l|}
$-6.463^{*}$ \\
\end{tabular} & 0.259 & 0.247 & $1.487^{* *}$ \\
\hline RIL A29 x RIL A5 & $0.684 * *$ & $0.782 * *$ & -0.013 & -2.406 & $0.301 * *$ & 0.090 & $19.426 * *$ & -0.320 & -0.197 & -0.081 \\
\hline RIL A30 x RIL A5 & -0.013 & 0.129 & 1.019 & 1.527 & -0.040 & -0.067 & $-19.796 * *$ & -0.092 & 0.120 & $1.270 *$ \\
\hline RIL A2 x RIL A10 & -0.191 & 0.241 & -1.491 & 0.788 & $-0.380 * *$ & $-0.256 *$ & $11.093 * *$ & -0.181 & $0.940 * *$ & -0.453 \\
\hline RIL A3 x RIL A10 & -0.108 & -0.094 & 0.226 & 2.390 & 0.166 & -0.110 & 4.426 & 0.217 & 0.171 & -0.939 \\
\hline RIL A7x RIL A10 & 0.023 & $0.620 * *$ & -1.580 & -1.760 & -0.204 & -0.016 & $7.426 *$ & -0.135 & -0.306 & -0.967 \\
\hline RIL A8 x RIL A10 & $0.524 * *$ & $0.647 * *$ & $2.598 *$ & $-5.338^{* *}$ & $1.095 * *$ & 0.059 & $16.426 * *$ & $0.353 *$ & $0.864 * *$ & $3.083 * *$ \\
\hline RIL A14 x RIL A10 & -0.184 & 0.077 & -0.522 & $-3.557 *$ & $-0.728 * *$ & -0.034 & $16.426 * *$ & $-0.352 *$ & -0.319 & -0.776 \\
\hline RIL A18 x RIL A10 & -0.251 & $-0.472^{*}$ & $2.302^{*}$ & $4.886^{* *}$ & $-0.760 * *$ & -0.071 & $-43.574 * *$ & $0.709 * *$ & $1.403 * *$ & 0.813 \\
\hline RIL A19 x RIL A10 & 0.034 & 0.161 & $-2.540 *$ & -1.460 & $-0.303 * *$ & -0.107 & $10.870 * *$ & $-0.404 *$ & $-1.088 * *$ & $-1.593 * *$ \\
\hline RIL A22 x RIL A10 & $0.428 * *$ & $0.423 *$ & $4.348 * *$ & $5.006 * *$ & $1.044 * *$ & 0.016 & $-15.796 * *$ & k $-0.733 *$ & 0.183 & $2.385^{* *}$ \\
\hline RIL A23 x RIL A10 & 0.153 & $-0.583 * *$ & -1.184 & 0.917 & -0.145 & 0.140 & $-17.463 * *$ & $0.811^{* *}$ & -0.096 & $-1.461^{* *}$ \\
\hline RIL A26 x RIL A10 & 0.260 & 0.138 & 1.617 & $5.383 * *$ & $0.911^{* *}$ & $0.213^{*}$ & $-10.241^{* *}$ & $-0.525 * *$ & $-0.555^{* *}$ & 0.196 \\
\hline RIL A29 x RIL A10 & 0.018 & $-0.863^{* *}$ & -1.361 & -1.060 & -0.135 & 0.144 & 3.981 & $0.423 *$ & -0.316 & -0.335 \\
\hline RIL A30 x RIL A10 & $-0.706 * *$ & -0.293 & $-2.413^{*}$ & $-6.194 * *$ & $-0.560 * *$ & 0.021 & $16.426 * *$ & -0.183 & $-0.882 * *$ & 0.046 \\
\hline RIL A2 x RIL A20 & $0.633 * *$ & 0.165 & $4.064 * *$ & $-4.874 * *$ & $0.810^{* *}$ & $0.230 *$ & $-10.963 * *$ & -0.156 & $-0.965 * *$ & $1.672 * *$ \\
\hline RIL A3 x RIL A20 & $0.333^{*}$ & $-0.570 * *$ & $-2.460 *$ & -0.417 & -0.135 & 0.065 & $9.370 *$ & $-0.458^{*}$ & $-0.407^{*}$ & 0.315 \\
\hline RIL A7x RIL A20 & -0.102 & 0.101 & $2.275^{*}$ & 0.417 & -0.145 & -0.030 & $-15.296 * *$ & -0.310 & $0.476 *$ & 0.270 \\
\hline RIL A8 x RIL A20 & $-0.598 * *$ & 0.264 & $-3.421 * *$ & $6.172 * *$ & $-0.779 * *$ & -0.099 & 3.370 & 0.054 & $-1.174 * *$ & $-1.930 * *$ \\
\hline RIL A14 x RIL A20 & $0.598 * *$ & $-0.393^{*}$ & -1.264 & $4.440 *$ & $0.915^{* *}$ & -0.095 & 3.370 & 0.340 & $0.676 * *$ & 0.862 \\
\hline RIL A18 x RIL A20 & 0.230 & $0.755^{* *}$ & $-3.590 * *$ & 1.597 & $0.380 * *$ & 0.011 & $23.370 * *$ & $0.374 *$ & $-1.452 * *$ & -0.400 \\
\hline RIL A19 x RIL A20 & $-0.571^{* *}$ & 0.062 & $4.455^{* *}$ & -2.600 & -0.114 & 0.135 & $-30.519 * *$ & -0.029 & $0.974 * *$ & $2.462 * *$ \\
\hline RIL A22 x RIL A20 & $-0.715^{* *}$ & $-0.376^{*}$ & $-4.004 * *$ & $-5.250 * *$ & $-1.180 * *$ & $0.238^{*}$ & $19.481^{* *}$ & 0.075 & $0.532 * *$ & $-2.027 * *$ \\
\hline RIL A23 x RIL A20 & $0.681 * *$ & $0.391 *$ & $2.770 * *$ & -2.422 & $0.767^{* *}$ & $-0.218^{*}$ & 1.148 & -0.330 & -0.244 & $1.359 *$ \\
\hline RIL A26 x RIL A20 & $-0.506^{* *}$ & $-0.645^{* *}$ & -1.592 & $-5.197 * *$ & $-0.953 * *$ & -0.048 & $16.704 * *$ & 0.266 & 0.307 & $-1.683 * *$ \\
\hline RIL A29 x RIL A20 & $-0.701 * *$ & 0.081 & 1.374 & $3.467 *$ & -0.166 & $-0.234 *$ & $-23.407^{* *}$ & -0.102 & $0.513 * *$ & 0.416 \\
\hline RIL A30 x RIL A20 & $0.719 * *$ & 0.164 & 1.395 & $4.667^{*}$ & $0.600 * *$ & 0.046 & 3.370 & 0.275 & $0.763 * *$ & $-1.316^{*}$ \\
\hline LSD $5 \%$ & 0.263 & 0.315 & 1.857 & 3.342 & 0.206 & 0.188 & 6.403 & 0.348 & 0.337 & 1.010 \\
\hline LSD $1 \%$ & 0.373 & 0.448 & 2.640 & 4.751 & 0.292 & 0.268 & 9.103 & 0.495 & 0.479 & 1.436 \\
\hline
\end{tabular}


positive specific combining ability effects for seed cavity diameter only, while it had highly significant negative specific combining ability effects for total yield and flesh thickness. The cross RIL A23 $\times$ RIL A20 showed highly significant positive specific combining ability effects for LAI, total yield and average fruit weight while it had significant positive specific combining ability effects for early yield and TSS, but it had significant negative specific combining ability effects for FSI. According to Duradundi et al. (2018) the earliness and the high yield are an important traits in vegetables like muskmelon. The SCA effects of LAI ranged from -0.834 in the cross RIL A23 $\times$ RIL A5 to 0.719 in the cross RIL A30 $\times$ RIL A20. Ten out of 36 crosses showed positive highly significant and significant SCA effects of LAI. Regarding early yield, the SCA effects ranged from -0.911 in the cross RIL A8 $\times$ RIL A5 to 0.782 in the cross RIL A29 $\times$ RIL A5. Nine out of 36 crosses showed positive highly significant and significant SCA effects of early yield. The best crosses had specific combining ability (SCA) for early yield were RIL A29 $\times$ RIL A5 (poor $\times$ poor), RIL A18 $\times$ RIL A20 (good $\times$ poor), RIL A3 $\times$ RIL A5 (good $\times$ poor), RIL A8 $\times$ RIL A10 (poor $\times$ good), and RIL A7 $\times$ RIL A10 (poor $\times$ good). As for total yield, the SCA effects ranged from -4.004 in the cross RIL A22 $\times$ RIL A20 to 4.455 in the cross RIL A19 $\times$ RIL A20. Eight out of 36 crosses showed positive highly significant and significant SCA effects of total yield. The best crosses had specific combining ability (SCA) for total yield were RIL A19 $\times$ RIL A20 (poor $\times$ poor), RIL A22 $\times$ RIL A10 (good $\times$ poor), RIL A2 $\times$ RIL A20 (poor $\times$ poor), RIL A23 $\times$ RIL A20 (poor $\times$ poor), and RIL A8 $\times$ RIL A10 (good $\times$ poor).

Concerning average fruit weight, the SCA effects ranged from -1.180 in the cross RIL A22 $\times$ RIL A20 to 1.095 in the cross RIL A8 $\times$ RIL A10. Twelve out of 36 crosses showed positive highly significant SCA effects of average fruit weight. The best crosses had specific combining ability (SCA) for average fruit weight were RIL A8 $\times$ RIL A10 (poor $\times$ good), RIL A22 × RIL A10 (good × good), RIL A26 × RIL A10 (poor $\times$ good), RIL A2 $\times$ RIL A20 (good $\times$ poor), and RIL A23 $\times$ RIL A20 (good $\times$ poor). With respect to TSS, the SCA effects ranged from -2.027 in the cross RIL A22 $\times$ RIL A20 to 3.083 in the cross RIL A8 $\times$ RIL A10. Seven out of 36 crosses showed positive highly significant and significant SCA effects of TSS. The best crosses had specific combining ability (SCA) for TSS were RIL A8 $\times$ RIL A10 (good $\times$ poor), RIL A19 × RIL A20 (good $\times$ poor), RIL A22 × RIL A10 (good $\times$ poor), RIL A2 $\times$ RIL A20 (good $\times$ poor), RIL A26 $\times$ RIL A5 (poor $\times$ good), RIL A23 $\times$ RIL A20 (poor $\times$ poor) and RIL A30 $\times$ RIL A5 (poor $\times$ good).

Comparing the general combining ability impacts (GCA) of the parents to their related crosses (SCA) denoting that the GCA impacts of the parents were not affected in the SCA impacts of the hybrids for some of the studied characters. Thus, in some cases, the crossing between good general combiners inbred lines cannot necessity 
lead to good specific combinations and the same was true for certain poor combinations that included one good combiner, while in some other cases, both good combiners could give preferable combinations. In some cases, when two poor combiners were crossed, best combinations were noted to be produced. This indicated the inconsistent expression of SCA effect in specific crosses irrespective of GCA effect of the parents. Similar results were reported by Brar and Sukhija (1977), Sidhu and Brar (1977), Gill and Kumar (1989), Guravet al.(2000) and Chaudharyet al. (2006). Likewise, that indicates wide diversity in the ability of the inbred to give hybrid vigor. Any arrange of combination between the parents might produce heterosis over the parents that could be refer to preferable dominant genes, over-dominance or epistatic action of genes. Based on the current results, it could be deduced that the production of hybrids depend on the parental behavior was not practically true and this may be due to the interaction between genes and the final outcome of gene action, which control in this trait. Such results were also reported by Dhaliwalet al. (2003)on tomato. The cross combinations that were noted as good specific combiners can be used as genetic resources for heterosis breeding or in getting preferable recombinants/segregants in next generations for such characters.

Also, Khalil et al. (2015) reported that general and specific combining ability act an essential role in breeding programs. General and specific combining ability are the major parameters for quick evaluation and genotypes examining in Line $\times$ Tester analysis. These studies aid in parents choosing and classification for their potential performance through various cross combinations.

\section{c. Genetic Components and Heritability Degrees :}

That is illustrated from the data in Table (9) that shows the estimates of genetic variance components, dominance degree and heritability. Lines showed higher variances than testers for all characters except marketable yield percentage.

The GCA and SCA variances showed wide range of variation for whole the studied traits. SCA variances were bigger than GCA variances for whole the studied traits except average fruit weight. The higher value of SCA variances denotes the superiority of non-additive gene action that requires maintenance of heterozygosity in the population. Similarly, dominant variance components were greater than the additive components for LAI, early yield, total yield, marketable yield percentage, netting percentage and TSS, while the additive variance components were greater than the dominant variance components for the rest traits. Identical findings were stated by Kitroongruang et al. (1992) who reported that additive variance was much larger than the dominance variance for FSI. Moreover, Feysian et al. (2009) found a predominance of additive impacts of average fruit weight in a diallel of local melon populations in Iran. Also, Arasimovitch (1934) reported that dominant variance was 
Table 9. Estimates of genetic variance components, dominance degree and heritability for some melon characters the in the open field of 2018summer season.

\begin{tabular}{|c|c|c|c|c|c|c|c|c|c|c|}
\hline Source of Variance & $\begin{array}{c}\text { Leaf } \\
\text { area } \\
\text { index }\end{array}$ & $\begin{array}{c}\text { Early yield } \\
\text { (Ton/feddan) }\end{array}$ & $\begin{array}{c}\text { Total yield } \\
\text { (Ton/feddan) }\end{array}$ & $\begin{array}{c}\text { Marketable } \\
\text { yield } \\
(\%)\end{array}$ & $\begin{array}{c}\text { Average } \\
\text { fruit } \\
\text { weight } \\
\text { (ca) }\end{array}$ & $\begin{array}{c}\text { Fruit } \\
\text { shape } \\
\text { index }\end{array}$ & $\begin{array}{c}\text { Netting } \\
(\%)\end{array}$ & $\begin{array}{c}\text { Seed } \\
\text { Cavity } \\
\text { diameter } \\
\text { (am }\end{array}$ & $\begin{array}{c}\text { Flesh } \\
\text { thickness } \\
\text { (cm) }\end{array}$ & $\begin{array}{c}\text { Total } \\
\text { soluble } \\
\text { solids } \\
\text { (on }\end{array}$ \\
\hline$\sigma^{2}$ Testers & 0.027 & 0.073 & -0.036 & 0.137 & -0.018 & 0.028 & 117.347 & 0.135 & -0.033 & 0.439 \\
\hline$\sigma^{2}$ inbred Lines & 0.360 & 0.535 & 8.318 & -0.520 & 0.048 & 0.148 & 299.480 & 1.047 & 0.471 & 2.624 \\
\hline$\sigma^{2} \mathrm{GCA}$ (average) & 0.128 & 0.005 & 0.067 & 0.003 & 0.663 & 0.002 & 3.731 & 0.010 & 0.003 & 0.026 \\
\hline $\begin{array}{c}\sigma^{2} \mathrm{SCA}(\text { Inbred Lines } \\
\mathrm{xTesters)}\end{array}$ & 0.296 & 0.305 & 7.064 & 16.370 & 0.517 & 0.014 & 429.748 & 0.242 & 0.631 & 2.186 \\
\hline Additive $\delta^{2} \mathrm{~A}$ & 0.513 & 0.721 & 3.286 & 0.013 & 0.151 & 0.066 & 514.036 & 1.040 & 0.514 & 0.505 \\
\hline Dominance $\delta^{2} \mathrm{D}$ & 1.186 & 1.221 & 18.256 & 65.479 & 0.034 & 0.054 & 880.300 & 0.969 & 0.426 & 1.915 \\
\hline $\begin{array}{c}\text { Variance ratio } \\
\left(\sigma^{2} \mathrm{GCA} / \sigma^{2} \mathrm{SCA}\right)\end{array}$ & 0.433 & 0.017 & 0.009 & 0.002 & 1.281 & 0.111 & 0.009 & 0.041 & 0.005 & 0.012 \\
\hline $\begin{array}{c}\text { Dominance Degree } \\
\left(\sigma^{2} \mathrm{~A} / \sigma^{2} \mathrm{D}\right)^{1 / 2}\end{array}$ & 0.658 & 0.768 & 0.424 & 0.014 & 2.107 & 1.102 & 0.764 & 1.036 & 1.098 & 0.514 \\
\hline$\left(\delta^{2} \mathrm{D} / 2 \delta^{2} \mathrm{~A}\right)^{0.5}$ & 0.551 & 0.663 & 5.477 & 0.649 & 0.051 & 0.042 & 475.661 & 0.710 & 0.331 & 0.695 \\
\hline $\mathrm{h}_{\text {Bs } \%}$ & 95.766 & 94.715 & 85.158 & 84.345 & 80.073 & 75.670 & 96.898 & 93.844 & 88.388 & 68.556 \\
\hline $\mathrm{h}_{N S} \%$ & 28.915 & 35.167 & 12.990 & 25.035 & 65.357 & 41.511 & 35.723 & 48.577 & 48.331 & 14.306 \\
\hline
\end{tabular}

greater than the additive variance for TSS and net appearance. So, inbred lines selection in advanced generations from the highly heterotic cross is suggested for improving these characters. That might be attributed to the fact that statistically GCA variance is the additive portion of variability but it also involves additive $\mathrm{x}$ additive and higher orders of epistatic interactions (Matzinger and Kempthorne, 1956).

The ratio of GCA/SCA variances were very higher than one for average fruit weight only that showed the superiority of additive gene action over the non-additive gene action for this character. In contrast, the ratio of GCA/SCA variances were much lower than one for whole the rest traits that explained the superiority of non-additive gene action over the additive gene action. The non-additive component of genetic variance had the major role in the inheritance of these traits. Previous studies on melon also indicated that the predominance of non-additive gene action for the majority of melon characters (Dhaliwaland Lai, 1996). Also, Khalil et al. (2015) reported the same result in tomato.

The estimates of dominance degree, which was less than one, also proved the additive action of genes for these characters. The role of additive gene action controlling these characters was reported on FSI (Kitroongruanget al.,1992) and average fruit weight (Feysian et al., 2009).

However, GCA variances were greater than SCA indicating the importance of additive genes more than non-additive genes that governing average fruit weight trait only, while the rest traits that their SCA variances were bigger than GCA denoting the more importance of non-additive genes versus additive genes. 
Determines of broad sense heritability $\left(\mathrm{h}_{\mathrm{BS}}\right.$ ) were great for whole the studied characters, since they ranged from $68.556 \%$ to $96.898 \%$ for TSS and netting percentage, respectively. The great BSH estimated denotes the minor role of the environment on these characters. Besides, narrow sense heritability values (hNs) ranged from 12.990 to 65.357 for total yield and average fruit weight, respectively. Also, the great NSH estimate denotes the importance of additive impact of genes governing these characters. These results are in partial agreement with those of Javanmard et al. (2018) who reported that narrow sense heritability was high for all melon traits except fruit diameter and TSS. In contrast, Mohammadi et al. (2014) stated that the broad and narrow sense heritability in melon were low for average fruit weight, flesh thickness and total yield, but they were high for TSS. These results indicate that selection may be more effective for improving traits of genotypes in early generations.

\section{d. The Contribution of Inbred Lines and Testers :}

The proportional contribution of inbred lines, testers and their interaction was displayed in Table (10). It is illustrated from the data in Table (10) that the proportional contribution of testers were high for most traits except marketable yield percentage and average fruit weight. However, the results showed lower contribution of inbred lines than the individual contribution of testers for all characters except marketable yield percentage and average fruit weight. Testers were more important for productive as shown for FSI (66.959\%), seed cavity diameter $(64.869 \%)$, and early yield $(49.714 \%)$ which illustrated superiority effect for these characters. The contribution of maternal and paternal interaction (Inbred line $x$ Tester) played important role towards some of the characters that was found to be higher than the individual contribution where it had high values for marketable yield percentage (63.564\%), average fruit weight (61.028\%), netting percentage $(36.743 \%)$ and flesh thickness $(43.818 \%)$, while the lowest proportional contribution of maternal and paternal interaction (Inbred line $x$ Tester) was shown in FSI and seed cavity diameter, Table 10.. Proportional contribution of Lines, Testers and (Lines $\mathrm{x}$ Testers) to the total variance for some melon characters in the open field of 2018 summer season.

\begin{tabular}{|c|c|c|c|c|c|c|c|c|c|c|}
\hline $\begin{array}{c}\text { Proportional } \\
\text { Contribution } \\
(\%)\end{array}$ & $\begin{array}{c}\text { Leaf } \\
\text { area } \\
\text { index }\end{array}$ & $\begin{array}{c}\text { Early yield } \\
\text { (Ton/feddan) }\end{array}$ & $\begin{array}{c}\text { Total yield } \\
\text { (Ton/feddan) }\end{array}$ & $\begin{array}{c}\text { Marketable } \\
\text { yield } \\
(\%)\end{array}$ & $\begin{array}{c}\text { Average } \\
\text { fruit } \\
\text { weight } \\
(\mathrm{kg})\end{array}$ & $\begin{array}{c}\text { Fruit } \\
\text { shape } \\
\text { index }\end{array}$ & $\begin{array}{c}\text { Netting } \\
(\%)\end{array}$ & $\begin{array}{c}\text { Seed cavity } \\
\text { diameter } \\
(\mathrm{cm})\end{array}$ & $\begin{array}{c}\text { Flesh } \\
\text { thickness } \\
(\mathrm{cm})\end{array}$ & $\begin{array}{c}\text { Total } \\
\text { soluble } \\
\text { solids } \\
(\%)\end{array}$ \\
\hline Tester (T) & 44.670 & 49.714 & 44.182 & 4.014 & 11.611 & 66.959 & 30.339 & 64.869 & 37.459 & 40.794 \\
\hline $\begin{array}{c}\text { Inbred Lines } \\
\text { (Il) }\end{array}$ & 21.326 & 22.701 & 18.444 & 32.423 & 27.362 & 22.249 & 32.918 & 19.233 & 18.724 & 25.515 \\
\hline (IL X T) & 34.005 & 27.585 & 37.374 & 63.564 & 61.028 & 10.793 & 36.743 & 15.898 & 43.818 & 33.691 \\
\hline
\end{tabular}


indicating predominant maternal influence for these traits. These findings are coincided with those of Khalil et al. (2015) who stated that the contribution of maternal and paternal interaction (Inbred line $x$ Tester) played important role towards some of the characters that was found to be higher than the individual contribution in tomato.

As a conclusion, the used genotypes differed in significance indicating the presence of genetic differences among them. The significant heterotic crosses denoted predominance of non-additive gene action in genetic control of the studied traits. The cross combinations that were observed as good specific combiners (SCA) could be genetic resources for heterosis breeding to produce desirable recombinants and offsprings in the early segregating generations. The inbred lines RIL A 5 (T5) showed higher positive general combining ability (GCA) effect for all characters except early yield and FSI, which make it could be used as parent in breeding programs and the potential parent (good combiner) that in selection program would be effective for its efficient use in subsequent crossing programs for more LAI, total yield, marketable yield percentage, average fruit weight, netting percentage, flesh thickness, TSS and less seed cavity diameter. The best specific combining ability (SCA) was observed in hybrids RIL A29 × RIL A5 for early yield, RIL A19 × RIL A20 for total yield and RIL A8 $\times$ RIL A10 for average fruit weight and TSS. The good specific combiners could be used as genetic materials in heterotic breeding programs for producing new hybrids with desirable characters.

\section{REFERENCES}

1. Arasimovitch, V.V. 1934. Inheritance of sugar content in cucurbits. Bull. Appl. Bot. Genet. Plant Breeding, 5: 5-31.

2. Bitzer, M.L.; F.L. Patterson and W.E. Nyquist. 1967. Diallele analysis and gene action in crosses of Triticum aestivum. L. Agron. Abstr., Medison, pp.4.

3. Brar, J.S. and B. S. Sukhija. 1977. Line $x$ tester analysis for combining ability in watermelon (Citrullus lanatus Thunbs. Mansf.). Indian J. Hort., 34: 410-414.

4. Chadha, M.L. and K.S. Nandpuri. 1980. Hybrid vigour studies in muskmelon. Indian J. Hort, 37 (3): 276-282.

5. Chandha, S.; J. Kumarand Vidyasagar. 2001. Combining ability over environments in tomato. Ind. J. Agric. Res., 35(3):171-175.

6. Chaudhary, B.R.; R.S. Dhaka and M.S. Fageria. 2003. Heterosis for yield and yield related attributes in muskmelon (Cucumis melo L.). Indian J. Gen., 63(1): 91-92.

7. Chaudhary, B.R.; M.S. Fageria; S. Pandey and M. Rai. 2006. Combining ability studies for economic attributes in muskmelon (Cucumis melo L.). Veg. Sci., 33(2): 185-187. 
8. Chaudhary, B. R. and S. Pandey. 2010. Breeding of F1 hybrids in muskmelon: Accomplishment and prospects. Indian Journal of Arid Horticulture 5 (1-2): 1-5.

9. Dhaliwal, M.S. 1997. Heterosis breeding in muskmelon-are view. Agricultural Reviews. 18 (1):35-42.

10. Dhaliwal, M.S. and T. Lai. 1996. Genetics of some important characters using line $X$ tester analysis in muskmelon. Indian J. Genet, 56(2) : 207-213.

11. Dhaliwal, M.S.; S. Singh and D.S. Cheema. 2003. Line $x$ Tester analysis for yield and processing attributes in tomato. J. Agric. Res. 40(1): 49-53.

12. Dixit, J. and G. Kalloo. 1983. Heterosis in muskmelon (Cucumis melo L.). J. Res. Haryana Agric. Univ., 13(4): 549-553.

13. Duradundi, S.K.; V.D. Gasti; R. Mulge; M.G. Kerutagiand D.A. Masuthi. 2018. Heterosis studies in muskmelon (Cucumis melo L.) for growth, earliness and yield traits. International Journal of Chemical Studies, 6(4): 3079-3086.

14. Falconer, D.S. and T.F.C. MacKay. 1996. Introduction to quantitative genetics.4th edition. Longman Green, Harlow, Essex, UK.

15. Feyzian, E.; H. Dehghani; A.M. Rezai and M.J. Javaran. 2009. Diallel cross analysis for maturity and yield-related traits in melon (Cucumis melo L.). Euphytica, 168: 215-223.

16. Gill, B.S. and J.C. Kumar. 1989. Combining ability analysis for fruit and quality characters in watermelon. Annals of Biology (Ludhiana)., 5(1): 49-53.

17. Gomez, A.K. and A.A. Gomez. 1984. Statistical Procedures for Agricultural Research. 2nd ed. John Wiley \& Sons Pub., New York.

18. Griffing, B. 1956. Concept of general combining ability in relation to diallel crossing system. Australian J. Bio. Sci., 9: 463-493.

19. Gurav, S.B.; K.N. Wavhal and P.A. Navale. 2000. Heterosis and combining ability in muskmelon (cucumis melo L.). J. Maharashtra Agric. Univ. (India).,25(2) : 149152.

20. Gusmini, G. and T.C. Wehner. 2008. Fifty-five years of yield improvement for cucumber, melon and watermelon in the United States. Hortechnology 18: 9-12.

21. Javanmard, T.; F. SoltaniSaleh-abadi and M.R. Bihamta. 2018. Estimation of some genetic parameters through generation mean analysis in melon. Indian $\mathrm{J}$. Agric. Res., A-355: 1-6.

22. Kalb, T.J. and D.W. Davis. 1984. Evaluation of combining ability, heterosis and genetic variance for fruit quality characteristics in bush muskmelon. J. Am. Soc. Hortic. Sci., 109(3): 411-415.

23. Kempthorne, O. 1957. An introduction of genetic statistics. John Willey \& Sons Inc. New York, USA pp: 468-473. 
24. Khalil, E.M; A.A. Farrag; A. A. Kheder and H. M. Mazyad. 2015. Breeding tomato hybrids for tomato yellow leaf curl virus (TYLCV) resistance and heat tolerance. Egypt. J. of Appl. Sci., 30 (9): 556-579.

25. Kitroongruang, N.; W. Poo-Swang and S. Tokumasu. 1992. Evaluation of combining ability, heterosis and genetic variance for plant growth and fruit quality characteristics in Thai-melon (Cucurnis melo L., var. acidulous Naud.). Scientia Horticulturae, 50: 79-87.

26. Koch, Gary, G. and P. K. Sen. 1968. Some aspects of the statistical analysis of the mixed mode.1. Biometrics 24: 27-48.

27. Lal, T. and R. Kaur. 2002. Heterosis and combining ability analysis for improvement horticultural traits and reduction to downy mildew in muskmelon (Cucumis melo L.). PAU Journal of Research, 39(4): 482-490.

28. Lippert, L.F. and P.D. Legg. 1972. Diallel analysis for yield and maturity characteristics in muskmelon cultivars. J. Am. Soc. Hort.Sci., 97: 87-90.

29. Matzinger, D. and O. Kempthorne. 1956. The modified diallel table with partial inbreeding and interaction with environment. Genetics, 41: 822-833.

30. Mishra, J.P. and V.S. Seshadri. 1985. Male sterility in muskmelon (Cucumis melo L.): II. Studies on heterosis. Genetica Agraris, 39 : 367-376.

31. Mohammadi, R.; H. Dehghaniand G. Karimzadeh. 2014. Genetic analysis of yield components, early maturity and total soluble solids in cantaloupe (Cucumis melo L. subsp. melo var. cantalupensis Naudin). YYU J. AGR. SCI. 24(1): 79-86.

32. Monforte, A.J.; M. Oliver; M.J. Gonzalo; J.M. Alvarez; R. Dolcet-Sanjuan and P. Arus, 2004. Identification of quantitative trait loci involved in fruit quality traits in melon (Cucumis melo L.). Theor. Appl. Genet., 108: 750-758.

33. More, T.A. and V.S. Seshadri. 1998. Improvement and cultivation of muskmelon, cucumber and watermelon. In: Cucurbits. Nayar, N.M. and More, T.A. (eds.). Oxford and IBH Publishing Co. Pvt. Ltd., New Delhi, pp. 169-186.

34. Munger, H.M. 1942. The possible utilization of first generation muskmelon hybrid and an improved method of hybridization. Proc. Amer. Soc. Hort. Sci., 40: 405410.

35. Munshi, A.D and V.K. Verma. 1997. Studies on heterosis in muskmelon (Cucumis melo L.). Vegetable Science, 24: 103-106.

36. Napolitano, M.; E. Jones-Evans; S. Krishnamurthyand E. Albertini. 2017. Exploring heterosis in melon (cucumis melo L.) Hybrids. Proceedings of the Joint Congress SIBV-SIGA, September, 2017, Pisa, Italy, pp. 19-22.

37. Pandey, S.; M. Rai and B. Singh. 2005. Genetic variability and character association in muskmelon (Cucumis melo L.). Indian J. Plant Genet. Resour., 18 (2):212-216. 
38. Peter, K.V. and K.R.M. Swamy. 2006. Advances in breeding of vegetables. In: Proceedings of II ${ }^{\text {nd }}$ National Plant Breeding Congress held at TNAU, Coimbatore from 1-3 March, 2006. Pp 1-23.

39. Pitrat. 2009. Melon. In: Vegetables I (Asteraceae, Brassicaceae, Chenopodiaceae and Cucurbitaceae). Edited by Prohens J. and Nuez F., Spinger International Edition, Spinger (India). Pp 283-316.

40. Randhawa, K.S. and M.J. Singh. 1990. Assessment of combining ability, heterosis and genetic variance for fruit quality characters in muskmelon (Cucumis melo L.). Indian J. Genet, 50 (2) . 127-130.

41. Rashidi, M. and K. Seyfi. 2007. Classification of fruit shape in cantaloupe using the analysis of geometrical attributes. World Journal of Agricultural Sciences 3 (6): 735-740.

42. Riggs,T.J. 1988. Breeding F1 hybrid varieties. J. Hort. Sci., 63:362-369.

43. Robinson, R. W.; H. M. Munger; T. W. Whitaker and G.W. Bohn. 1976. Genes for cucurbitaceae. Horticultural Science. 11:554-568.

44. Roy, N.C.; V.N. Jettopujov and N.M. Solanik. 2002. Combining ability for some agronomic characters in alfalfa (Medicago sativa L.).Pak. J. Agric. Res., 17(4): 346-350.

45. Sharif, A.; A. Bakhsh; M. Arshad; A.M. Haqqani and S. Najma. 2001. Identification of genetically superior hybrids in chickpea (Cicer arietinum L.). Pak. J. Bot., 33(4): 403- 409.

46. Sharma, H.R.; D. Sharma and A.K. Thakur. 2006. Studies on analysis of generic divergence in tomato (Lycopersicon esculentum L.). J. Hort. Sci. 1: 52-54.

47. Shashikumar, K.T. and M. Pitchaimuthu. 2016. Heterosis and combining ability analysis of quantitative and qualitative traits in muskmelon (Cucumis melo L). Int. J. Agaric. Sci. Res., 6 (2): 341-348.

48. Sidhu, A.S. and J.S. Brar. 1977. Heterosis and combining ability of yield and its components in watermelon (Citrullus lanatus (Thunb.) Mansf.). J. Res. (P.A.U)., 14(1): 52-58.

49. Singh, M.J. and K.S. Randhawa. 1990. Evaluation of heterosis and ability for traits in muskmelon. Indian J. Hort., 47:228-232.

50. Spargue, G.F. and L.A. Tatum. 1942. General versus specific combining ability in single crosses of corn. J. American Soc. Agron., 34: 923-932.

51. Subramanian, D. 2008. Studies on heterosis expression and association of fruit yield and yield component characters among five intervarietal crosses of Vellari melon (Cucumis melo L.). Madras Agricultural Journal, 95 (1-6): 24-31.

52. Wynne, J. C.; D. A. Emery and P. W. Rice. 1970. Combining ability estimates in Arachis hypogea L. II. Field performance of F1 Hybrids. Crop Sci., 10: $713-715$. 


\title{
قوة الهجين والقدرة على الإتتلاف لبعض صفات جودة تُمار سلالات الثمام المصرية المرباه ذاتياً بإستخدام تحليل Line X Tester.
}

\author{
محمد أبو الفتوح محمد سليم \\ قسم بحوث تربية الخضر والنباتات الطبية والعطرية - معهد بحوث البساتين - مركز البحوث الزر اعية - مصر \\ أجريت هذه الدر اسة فى مزرعة بحوث الخضر بقها بمحافظة القليوبية داخل جمهورية مصـر العربيـة \\ بالحقل المكثوف فى العروة الصيفية خلال الفترة من 2016 إلى 2018.حيث قُيمت ثلاثثن سلالة مـن الثنــمام

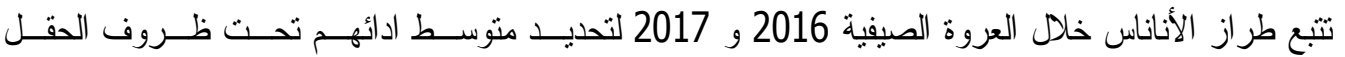 \\ المكثوف.
}

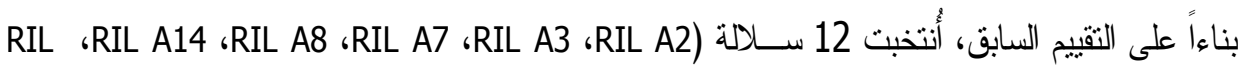

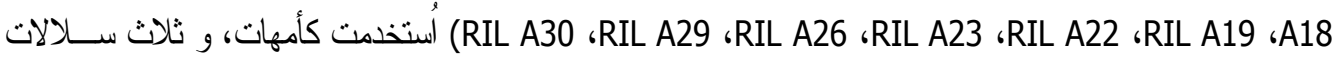

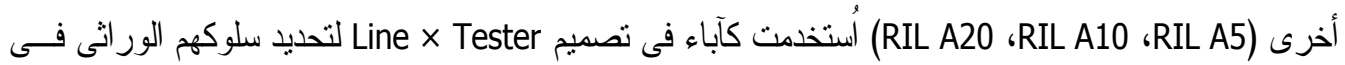

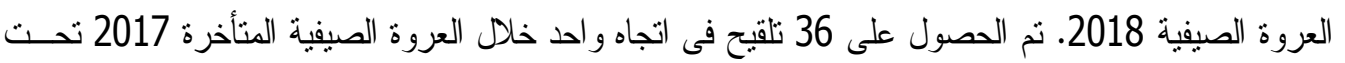

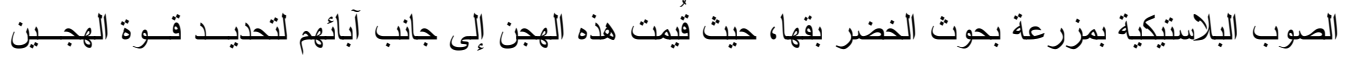

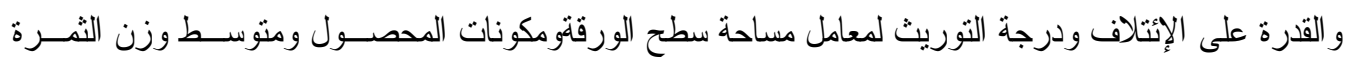
و النسبة المئوية للثبكة ومعامل شكل الثمرة وقطر فجوة البذور وسمك اللحم ونسبة المواد الصلبة الذائبة.

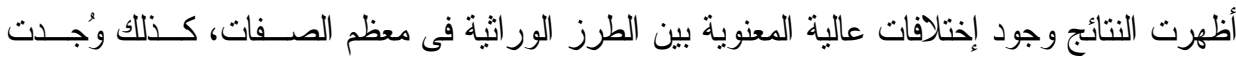

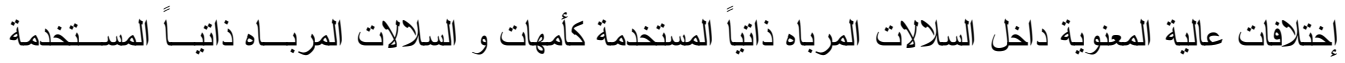

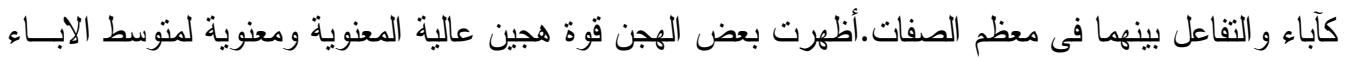
و لأفضل الاباء لمعظم الصفات.

أظهرت السلالة RIL A5 (T5) قدرة عامة على التآلف موجبة و مرتفعة لجميع الصفات باسنثناء صفتى

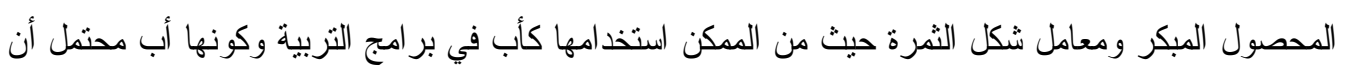

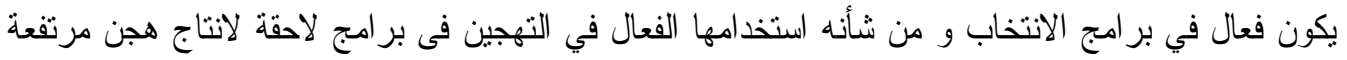

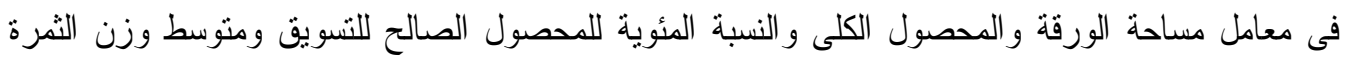
و النسبة المئوية للثبكة وسمك اللحم ونسبة المواد الصلبة الذائبة الكلبة وصغر قطر فجوة البذور .أظهرت تشعة ولهية وثمانية واثنى عشرة وسبعة هجن قدرة خاصة على التآلف تتكرج من عالية المعنوية إلى المعنوية فى صفات

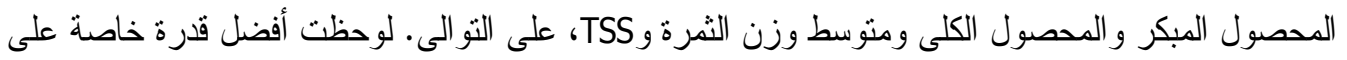

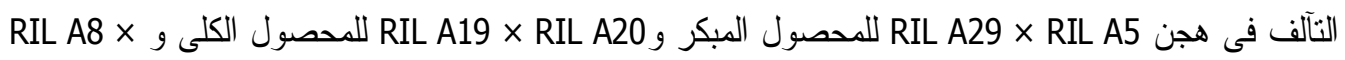

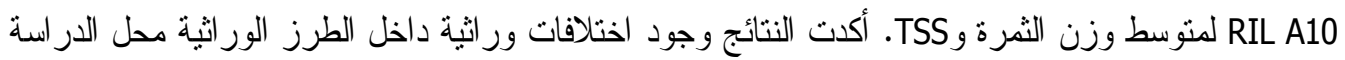
(الآباء الدذكرة و المؤنثة) كما الثارت قوة الهجين فى تلك الهجن الى وجود سيادة لفعل الجين غير المضيف في 